\title{
Chiral peculiar properties of self-organization of diphenylalanine peptide nanotubes: modeling of structure and properties
}

\author{
Bystrov V.S. ${ }^{* 1}$, Zelenovskiy P.S. ${ }^{2,3}$, Nuraeva A.S. ${ }^{2}$, Kopyl S. ${ }^{3}$, \\ Zhulyabina O.A. ${ }^{4}$, Tverdislov V.A. ${ }^{4}$ \\ ${ }^{1}$ Institute of Mathematical Problems of Biology - the Branch of the Keldysh Institute of \\ Applied Mathematics of the RAS, Pushchino, Moscow region, Russia \\ ${ }^{2}$ School of Natural Sciences and Mathematics, Ural Federal University, Ekaterinburg, Russia \\ ${ }^{3}$ CICECO-Aveiro Institute of Materials, University of Aveiro, Aveiro, Portugal \\ ${ }^{4}$ Faculty of Physics, Lomonosov Moscow State University, Moscow, Russia
}

\begin{abstract}
The structure and properties of diphenylalanine peptide nanotubes based on phenylalanine were investigated by various molecular modeling methods. The main approaches were semi-empirical quantum-chemical methods (PM3 and AM1), and molecular mechanical ones. Both the model structures and the structures extracted from their experimental crystallographic databases obtained by $\mathrm{X}$-ray methods were examined. A comparison of optimized model structures and structures obtained by naturally-occurring self-assembly showed their important differences depending on D- and L-chirality. In both the cases, the effect of chirality on the results of self-assembly of diphenylalanine peptide nanotubes was established: peptide nanotubes based on the D-diphenylalanine (D-FF) has high condensation energy $E_{0}$ in transverse direction and forms thicker and shorter peptide nanotubes bundles, than that based on L-diphenylalanine (L-FF). A topological difference was established: model peptide nanotubes were optimized into structures consisting of rings, while naturally self-assembled peptide nanotubes consisted of helical coils. The latter were different for the original L-FF and D-FF. They formed helix structures in which the chirality sign changes as the level of the macromolecule hierarchy raises. Total energy of the optimal distances between two units are deeper for L-FF $(-1.014 \mathrm{eV})$ then for D-FF $(-0.607 \mathrm{eV})$ for ring models, while for helix coil are approximately the same and have for L-FF $(-6.18 \mathrm{eV})$ and for D-FF $(-6.22 \mathrm{eV})$ by PM3 method; for molecular mechanical methods energy changes are of the order of $2-3 \mathrm{eV}$ for both the cases. A topological transition between a ring and a helix coil of peptide nanotube structures is discussed: self-assembled natural helix structures are more stable and favourable, they have lower energy in optimal configuration as compared with ring models by a value of the order of $1 \mathrm{eV}$ for molecular mechanical methods and $5 \mathrm{eV}$ for PM3 method.
\end{abstract}

Key words: diphenylalanine, peptide nanotube, molecular modeling, semi-empirical methods, DFT, ab initio methods, molecular mechanics, chirality, topology, self-assembly.

\section{INTRODUCTION}

The self-arrangement of matter and self-assembly of a complex of biomolecular structures is one of the most important and intriguing phenomena in animate and inanimate nature, and 
is constantly in the focus of attention of researchers in various directions [1-5]. Of no less interest is computer assisted molecular modelling of the self-organization processes of molecular systems at different levels and by different methods [6-10].

One example of such self-organizing macromolecular systems are peptide nanotubes (PNT) based on the amino acid phenylalanine (Phe or F) and its dipeptide - diphenylalanine (FF, in their usual aquatic zwitterionic form H-FF-OH) [11-19]. Possessing a wide range of useful properties, these structures are promising for various applications in nanotechnology, nanoelectronics and biomedicine $[14,16]$. Self-assembly of such PNT occurs in aqueous media rather quickly and under certain conditions that affect their growth rate, the shape of self-organizing structures (their thickness and length) and their physical properties [20].

It should be noted that the role of symmetry and asymmetry [21-23], which are manifested here, in particular in such an important property as chirality $[2,24,25]$ of the original molecules, plays here a key role in the physical laws and processes at various levels of the organization of these structures [26-31]. Moreover, this is generally characteristic of the whole self-organization of matter and complex molecular systems [1, 2, 21-24].

The chirality of molecules has long been known. Thus, it was found that substances with the same chemical formula can rotate the plane of polarized light in different ways; they were called stereoisomers $[25,32]$. The two forms of stereoisomers are mirror reflections, but they are not combined with each other in space, like our left and right hands. This property of molecules is called chirality. At present, this term "chirality" (from ancient Greek "Xeı $\rho$ " hand) determines the property of a molecule not to be combined (coincided or superimposed) in space with its mirror image. More strictly mathematically: chirality is the incompatibility of an object with its mirror reflection for any combination of rotations and displacements in three-dimensional space [25]. Such mirror-symmetric forms (or stereoisomers) of chemical compounds are called enantiomers [33]. These are the "left" ( $\mathrm{L}$ - from the Latin "laeva") and the "right" (D - from the Latin "dextra") enantiomers. These two forms of enantiomers (or optical isomers) rotate the plane of polarized light either to the right (D) or to the left (L).

Modern advances in molecular biology and biophysics have already allowed a sufficiently detailed description of the structures and functions of almost all the major biomacromolecules at different levels of their hierarchy and structural organization [24-31]. A huge number of chiral molecules, such as amino acids, sugars, etc., exist in nature and play a crucial role in the living organisms [2]. At the same time, self-organizing macromolecules tend to form hierarchical structures with alternating signs of chirality upon transition to a higher hierarchical level [28-30].

Depending on the conformation ( $\alpha$-helix and $\beta$-sheet) and the type of stereoisomer ( $\mathrm{L}$ or D) of the primary structure (amino acid), the properties of any material assembled on their basis are also changing. At the same time, it is known that the characteristic feature of all bioorganic molecular systems in living organisms is the chiral specificity of their structures and functions. That is, from the point of view of chirality, all biopolymers have one common property here: the nucleotide units of RNA and DNA have only D-configuration, and the macromolecules of proteins and enzymes in all living organisms consist of only L-amino acids. In other words, DNA and proteins are homochiral polymers of the opposite chirality. Thus, such chiral specificity is an inherent property of living nature, and the reproduction and maintenance of such specificity is one of the most characteristic functions of life activity. This property, called homochirality, turns out to be an important position that underlies a common physical factor leading to hierarchical structure formation in proteins and nucleic acids [2830].

Hence, homochirality is one of the main properties of all biological macromolecules, with virtually no exceptions. Nevertheless, it turned out that in nature there are still "right" Damino acids. Thus, it was revealed [34] that D-amino acids play an important role in the life of some bacteria, controlling their reaction to various food stimuli and such a process as the 
"assembly" of the cell wall. Perhaps, this is a small exception. But they are important, especially for possible practical applications of the peptide structures of different chirality: on the basis of L-amino acids, and on the basis of D-amino acids.

And here an important point is the fact that life always uses only one of the enantiomers. If, for example, milk existed, the proteins of which consisted exclusively of D-amino acids, our organism could hardly digest it, since all the digestive enzymes are based on L-amino acids. Just because of this that it is extremely important for chemists to be able to separate enantiomers - indeed, different enantiomers of the same substance sometimes have a completely different effect on the organism. Thus, chemists themselves often refer to enantiomers as a single compound, since their chemical properties are identical. However, their biological activity can be completely different. "Right" and "left" drugs, interacting with chiral molecules in the body, such as enzymes, can act differently. The need for optically pure enantiomers is also explained here by the fact that often only one of them has the desired therapeutic effect, whereas the second antipode can be useless at best and, at worst, cause undesirable side effects or even be deadly toxic [35]. In order to avoid such "side effects", at present, attempts are made to produce many drugs in the form of optically pure compounds. However, obtaining them is difficult and expensive. Therefore, it is not surprising that among the multitude of the synthetic chiral preparations produced worldwide, only a small part is optically pure (that is, it consists of the molecules of the same chirality), while the rest ones are the racemates, that is, a mixture of molecules of both types of chirality.

It should be reiterated that an important and basic fact is that the overwhelming majority of the peptides in our living organisms are built on the basis of the "left" L-amino acids. The causes of such homochirality are a separate issue.

Usually, the classical mechanisms proposed here for the transition from a racemic mixture to homochiral biochemistry in the evolution are randomly attributed to the choice of L-amino acids and D-sugars by self-replication systems $[2,31]$. However, it is possible that there exists a physical factor (such as the effect of a weak neutral current [36]), which violates the equilibrium, which leads to the energy difference between one chiral molecule and its mirror isomer. This leads to a slight stabilization of L-amino acids and L-peptides (in the conformation of the $\alpha$-helix, $\beta$-sheet) relative to the corresponding enantiomer [36]. The energy difference may be enough here to break the chiral symmetry of the sequence of autocatalytic racemic reactions in an open nonequilibrium system [31]. And then, autocatalytic reactions can unwind the turns of asymmetry and chiral structures. This is a general hypothesis. However, it turned out that there are specific factors.

It is known that in physics, chirality is a property of elementary particles, consisting in the difference between right and left, and indicating that the Universe is asymmetric with respect to the replacement of the right by the left, and the left by the right. This symmetry breaking is already apparent at the levels of weak interactions [37, 38]. Thus, weak nuclear interaction is the only force of nature that has "preferences" in orientation: electrons emerged in a subatomic process, known as $\beta$-decay, are always left-sided. This means that their spin, the quantum angular momentum of elementary particles, will always be opposite to the direction of the electron's motion - these are the "left" spin-polarized electrons [39, 40].

In this regard, we note that among possible explanations of the reasons for the homochirality of biomacromolecules, there is a hypothesis by Wester - Ulbricht that the homochirality of life is a consequence of the spatial asymmetry of a weak interaction of elementary particles. They suggested that photons generated by these spin-polarized electrons could destroy more molecules of some type than those of the other, creating an imbalance. At the same time, it is possible that the spin-polarized electrons themselves can be the source of such asymmetry. Thus, experimental results published recently [41] prove the reality of at least one important element of this hypothesis: slow polarized electrons are indeed capable of initiating chirally selective chemical reactions in the gas phase [41, 42]. And, perhaps, as 
these studies with polarized electrons show, this effect is the source of the accumulated fact: the predominant existence in living organisms of "left" amino acids and "right" DNA molecules.

An important point is the influence of the magnetic field on the spin orientation of the electron and its directional motion in a magnetic field. Note that such a spin polarization of electrons by a magnetic field with synchrotron radiation was predicted as far back as 1963 $[43,44]$. The action of the magnetic field leads to the fact that the initially unpolarized electron beam, after some time, passes into a polarized state - with a spin directed opposite to the magnetic field, which is an energetically more favourable state. One of the important practical applications of this approach may be the possibility of separating a mixture of the chiral molecules into the enantiomers using spin-polarized electrons [45, 46]. Until now, only the methods based on different interactions of the enantiomers of some molecules with the enantiomers of other molecules have existed. In a recent paper [47] it was shown that enantiomers can be separated by selective adsorption to a magnetized surface, which occurs precisely because of the different spin polarization of electrons in enantiomers.

Another important question: what happens to the homochiral objects themselves? Here, with further hierarchical complication of molecular structures and transition to a still higher level of organization, a certain systemic molecular-biological pattern arises: there is a tendency of alternation of the sign of chirality of molecular structural levels of L-D-L-D for proteins and in a different order D-L-D-L for DNA [28-30]. That is, a periodic system of chirality is seen for various biomolecular elements [48-50]. In this case, the spirality (helicity) of the structures acts as a special case of chirality. This is the most important new approach to the modern analysis of various alternating chiral structures [28]. A detailed substantiation of these provisions was carried out recently in [28-30, 48-50], where a large amount of work was done on the analysis of helical and supercoiled structures in proteins and nucleic acids based on the PDP and CC + database [50]. The development of these works is in progress now.

In the field of materials science, chirality and supra-molecular organization have a great influence on the development of new nonlinear optical materials providing second-order nonlinear optical susceptibility, approximately 30 times greater than that of racemic material with the same chemical structure [38]. Chirality has also become an important concept in spintronics, where the chiral induced effect of spin selectivity is used to control electron spins transmitted through short organic molecules $[39,40]$ and long supramolecular structures [4547]. Some attempts are also known to create new chiral materials based on the chiralmagnetic effect [51], which is also associated with spin-polarized electrons and their behavior in a magnetic field $[43-46,52,53]$. This can be observed in the so-called three-dimensional Dirac semimetals [54]. On this basis, the possibilities of creating and applying materials with a given chirality are also being studied.

Apart from the influence of magnetic fields on chiral structures, the influence may also be exerted by electric fields, especially with a complex inhomogeneous (dipole and multipole) structure. Here an important observation was recently made in [55], in which it was shown that electrostatic interactions may be responsible for the chiral recognition and selection of chiral molecules. In this work, chiral recognition and chiral selection of individual enantiomers was observed during the self-assembly of two enantiomeric macroanions. It has been shown that individual enantiomers can be separated from their racemic mixtures by the introduction of chiral anionic molecules. The fact that relatively simple inorganic macro-ions demonstrate chiral recognition and selection during their self-assembly process indicates that similar features of biomacromolecules may be due to their macroionic character through longrange electrostatic interactions.

This means that there are features of electrical properties (distribution of charges and dipole moments) of molecules of different chirality, which determine the effect of electrostatic 
interactions [56] on their self-organization and the formation of structures in the process of self-assembly at different distances between these different chiral molecules, which can lead to different forms of the final structural formation. The practical application of these properties of the chirality of macromolecules requires a detailed study of the effects of their interaction depending on the type of chirality in various chiral molecular systems. Similar processes of self-sabotage, leading to the formation of individually different complex supramolecular peptide L- and D-structures, based on initially different, simpler left and right molecules, can be observed for polypeptides based on different amino acids having different dipole moments $[4,57-60]$. These studies can be effectively carried out using molecular computer simulation methods $[18,19]$ and be accompanied by experimental observations by atomic force and piezo-response force microscopy (AFM/PFM) [16, 17].

In this paper, we construct and investigate molecular models of molecular complexes of the type of diphenylalanine-peptide nanotubes (FF PNT) with different chirality (L-FF and DFF), features of their self-assembly, in comparison with the known experimental data. Despite numerous studies on the self-assembly of FF, their physical properties and applications, the role of chirality in their structure and properties are not well understood [11-20, 61-67]. Most of the work here was performed earlier on L-FF, starting with the original work by Görbitz [12], where the crystal structure of $\alpha$-helical L-FF PNT was determined. On the crystallographic structure of D-FF PNT, only recently experimental data have appeared [68, 69]. And we use them now in this work, as well as the data [12].

When modeling FF PNT, the chirality of F and FF, as well as the way amino acids are laid down when assembling nanotubes, are of great importance and affect their physical properties. For example, in [70] it was shown that the magnitude of the dipole moment and the polarization of the entire nanotube vary with their chirality. The purpose of this work is to substantiate and study the peculiarities of the effect of chirality on the structure and properties of such nanotubes using computer molecular modeling.

\section{COMPUTATIONAL DETAILS}

\subsection{Initial models and main computational details}

The initial molecular models of phenylalanine (commonly referred to as Phe or F) are taken from a special amino acid database that is included in the HyperChem software [71]. Then the molecular structure of the model of diphenylalanine (FF) was built in a special "working space" of the HyperChem software, and translated into a zwitterionic form, which is necessary for further modeling in accordance with experimental data $[18,19,70]$. For this purpose, in this paper we use the alpha-helix conformation of the initial amino acid $F$ and both its isomers of chirality L-F and D-F. Based on them, we create further models of dipeptides - diphenylalanine (FF) with various chiralities. For these two models, referred to as L-FF and D-FF, all necessary parameters were calculated using the PM3 quantum semiempirical method [71-73] in the restricted (RHF) and unrestricted (UHF) Hartree-Fock approximations [71-75]. After the initial quantum calculation, geometrical optimization was performed for both models using the Polak-Ribiere conjugate gradient method from the HyperChem [71] software. In the course of this optimization, the total energy of the system is calculated at each point, depending on the coordinates of all the atoms of the system, and the so-called potential energy surface (PES) [71] is determined, on which the minimum of this total energy or PES is found, corresponding to the optimal position of all the atoms of the system.

Optimized L-FF and D-FF models are shown in Figure 1. The main characteristics obtained for these models (shown in Fig. 1) are that they differ from each other in the orientation and values of their full dipole moment (in Debye units): see Table 1. Total energy of optimized nanostructures L-FF and D-FF, as well as the full dipole moments are about the 
same, but the orientations of their dipole moments, which are determined by the components Dy and Dz, turn out to be different and the values of these components alter between them. This distinction will play an important role in the following structural compositions for the self-organization of L-FF and D-FF molecules into more complex structures and their transformation into model structures, such as rings and nanotubes, in accordance with experimental data.

To obtain a better proof of the results, we used both ab initio and density functional theory (DFT) methods [76-82], which are accessible in the HyperChem package [71]. For the most in-depth confirmation of the calculated data, we compare our calculations performed using PM3 semi-empirical quantum methods with ab initio and DFT using the 6-31G* basis set with «385 basic Orbitals, 724 primitive Gaussians» [83-85], the MP2 correlation [86, 87] was also taken into account, and the HCTH98 approach [88] was used for the DFT calculation. All the methods are from the HyperChem software package [71]. The data obtained as a result of these calculations, presented in a separate paper [89], show close values of the dipole moment and polarization for both the structures. They have good convergence and are comparable when using different approaches and calculations using both ab initio, DFT, and semiempirical PM3 methods. Consequently, the PM3 method can be adequately used for further calculations for these molecular FF systems, and also for more complex structures based on them. The features of PM3 are discussed in [89-97], while PM3 is often used to calculate precisely organic molecules [71-73]. Main data for PM3 are presented in Table 1.
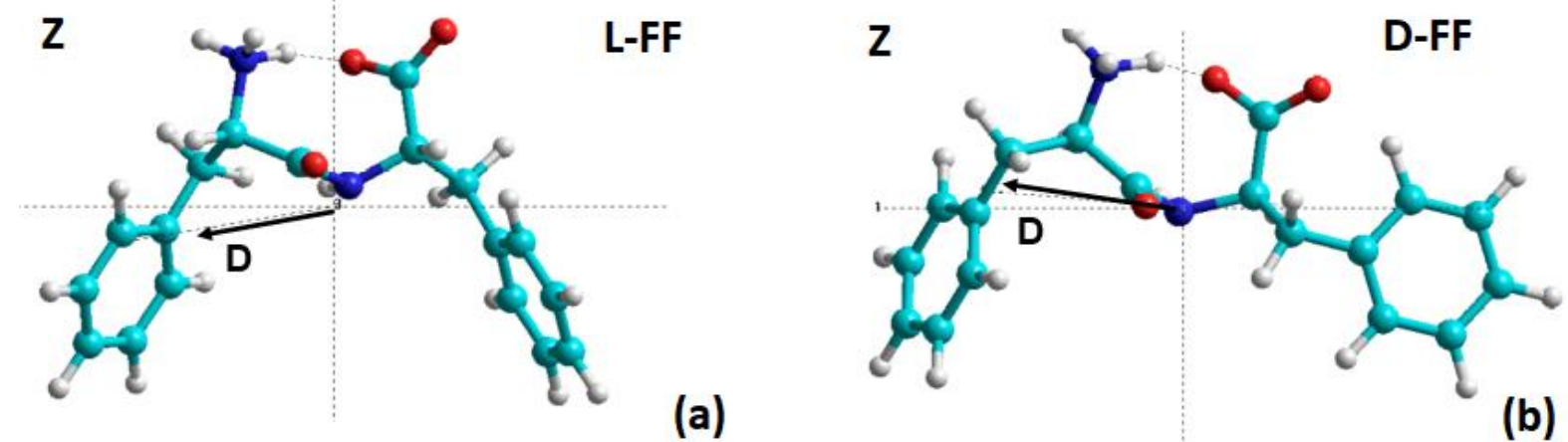

Fig. 1. Molecular models of diphenylalanine ( $\alpha$-helix conformation) in various isomer form: a) L-FF with chirality L, b) D-FF with chirality D. Dashed lines indicate the hydrogen bond N H ... O - (red - O, dark blue $-\mathrm{N}$, light blue $-\mathrm{C}$, gray $-\mathrm{H}$ ).

Table 1. Data computed for dipeptides L-FF and D-FF by PM3 method. Dipeptides consist from 43 atoms and have volume on the Van der Waals surface: $V_{\mathrm{L}-\mathrm{FF}}=291.540 \AA^{3}, V_{\mathrm{D}-\mathrm{FF}}-291.977 \AA^{3}$

\begin{tabular}{|l|c|c|c|c|c|c|c|c|}
\hline \multicolumn{1}{|c|}{ Method } & $\begin{array}{c}\text { FF } \\
\text { chirality }\end{array}$ & $\begin{array}{c}D_{\mathrm{x}}, \\
\text { Debye }\end{array}$ & $\begin{array}{c}D_{\mathrm{y}}, \\
\text { Debye }\end{array}$ & $\begin{array}{c}D_{\mathrm{z}}, \\
\text { Debye }\end{array}$ & $\begin{array}{c}D_{\mathrm{tot}}, \\
\text { Debye }\end{array}$ & $\begin{array}{c}\text { Polarization, } \\
\mathrm{C} / \mathrm{m}^{2}\end{array}$ & $\begin{array}{c}\text { Total energy, } \\
\text { a.u. }\end{array}$ & $\begin{array}{c}\text { RMS } \\
\text { gradient }\end{array}$ \\
\hline $\begin{array}{l}\text { PM3, both } \\
\text { RHF and UHF, } \\
112 \text { orbitals }\end{array}$ & L-FF & -10.507 & -1.784 & 0.834 & 10.69 & 0.122 & -133.962554 & $\sim 0.07$ \\
\cline { 2 - 8 } & D-FF & -11.63 & 1.052 & 1.113 & 11.73 & 0.134 & -133.959162 & $\sim 0.07$ \\
\hline
\end{tabular}

Computational molecular modeling of more complex tubular self-assembled nanostructures based on these two L-FF and D-FF dipeptides with different chirality was performed in this work using HyperChem software, similar to our previous studies [18, 19, 70]. But as distinct from work [70] in this article, we consider only the $\alpha$-helix conformations of the original amino acid molecules of phenylalanine (F) (since experimental data show that in crystallographic structures obtained by X-ray diffraction for these peptide nanotubes (PNT), no $\beta$-sheet conformation was found, namely, only $\alpha$-helix conformations are present in all the studied cases $[12,68,69,98]$. We used here chiral isomers L and D, for L-FF and DFF peptide nanotubes (PNT) structure models. For all FF PNT, we used zwitterionic 
molecular forms and considered, first, ring FF models consisting of 6 FF molecules, and, second, our earlier developed standard 2-ring models of tubular PNT, consisting from $6 \mathrm{FF}$ molecules in each ring, forming in total the hexagonal crystallographic structure in accordance with known experimental data [12, 18, 19, 68-70].

\subsection{Simulation and calculations of the properties of FF PNT nanotubes}

After optimization for both molecular models of L-FF and D-FF, their models are in the form of one ring (containing 258 atoms), and consisting of $6 \mathrm{FF}$ molecules (43 atoms for each individual structural segment - dipeptide FF molecules), as well as tubular structures in the form of 2 rings (out of 516 atoms) were built for the cases of L and D chirality. For all the cases, geometrical optimization was performed using the Polak-Ribiere conjugate gradient method [71] and optimized full structures of the L-FF and D-FF PNT nanotube models were obtained, similarly to what we had done earlier $[18,19,70]$.

The calculations were performed using the PM3 quantum semi-empirical method using various Hartree-Fock approximations (both RHF and UHF), and in some cases the ZINDO-1 [71] quantum semi-empirical method was also used. Here, in some cases, to refine the calculation results at some points, when poor convergence of calculations in the PM3 method occurred, similar methods AM1 [71, 99] (also included in the HyperChem package [71]) were also used. It is also known that, in fact, the PM3 method is a reparameterization of the AM1 method, which is based on the "neglect or ignoring the diatomic overlap differential (NDDO)" approach [71, 93-97]. PM3 differs from AM1 only in parameter values. The parameters for PM3 were obtained from a comparison of a much larger amount and a wider range of experimental data when compared with the calculated molecular properties. PM3, developed by Stuart [71-73], is based on the so-called "neglect of diatomic differential overlap (NDDO)" approximation, with several modifications and the choice of a wide range of parameters to reproduce the experimental values. Calculation methods with a higher level of accuracy (such as ab initio and DFT) usually require a long calculation time and a large amount of computer memory. PM3, as a rule, is here one of the most accurate and at the same time fast quantum computational methods, which uses several experimental parameters to simplify the computation process [73-75, 93-97]. Therefore, we tried to compare our calculated data for simpler di-peptide amino acid molecules by different high-precision methods with a faster and simpler semi-empirical quantum-chemical method, such as the PM3 approximation, in order to apply it already to our more complex and larger molecular self-assembled tubular and cluster structures. In addition, to calculate the energy change with variation of the distance between two different interacting structures based on FF, that is energy profiles (containing many steps of long calculations when changing the distances between interacting rings of different FF structures), molecular mechanics (MM) methods, such as MM+ (or MM2) [71, 100], Amber [101-103] and BIO CHARM [104] from the HyperChem package [71] were used. Comparison of the data calculated by these methods showed their good agreement with each other, and this confirmed the accuracy of the data obtained by all the methods.

After optimization, the values of the minimum total energy for all the systems and models of 2 FF rings (Fig. 2 with data from [70]) were determined, which correspond to the optimal distances between the rings, which was $\mathrm{d} \sim 5.45 \AA$. This also corresponded well to the experimental crystallographic data $([12,68,69])$, with similar small deviations between the structures of different chirality L-FF and D-FF.

The important result after these optimization processes is that shapes and configurations of both optimized structures are not equivalent, neither are the total dipole moment and energy. This result is due to the differences of the initial optimized states and configurations of L-FF and D-FF dipeptide molecules, which have different values and orientations of their dipole moment. As a result, the following electrostatic interaction has some individual 
peculiarities and variations for these two dipeptide molecules with different chirality. This could be considered as some common and similar features of long electrostatic interactions chiral molecules $[55,56]$.

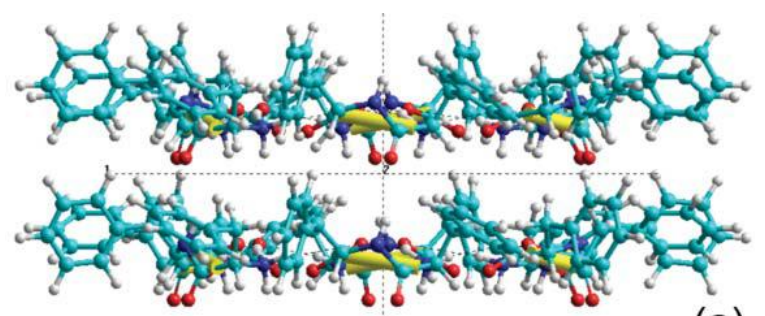

(a)
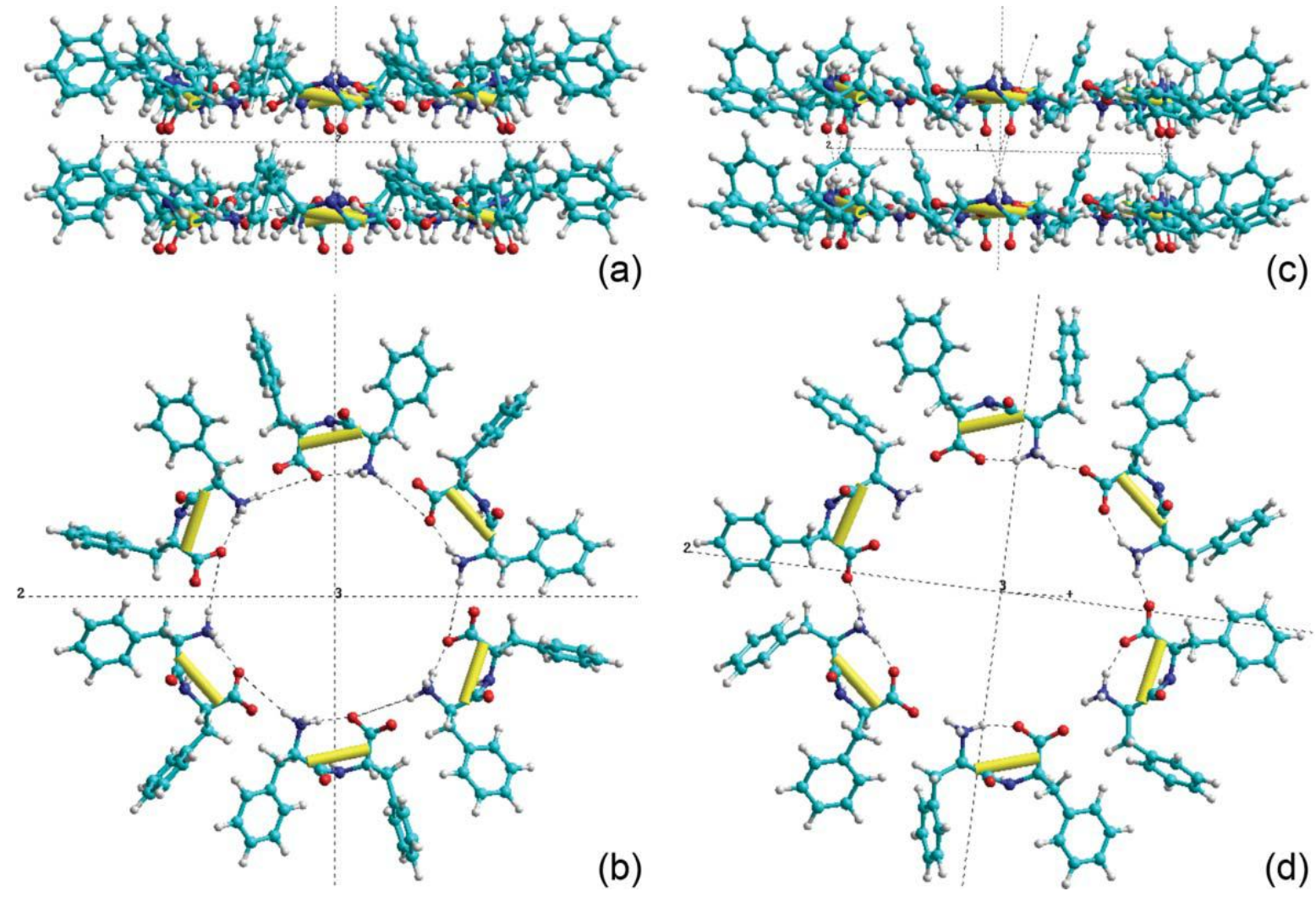

(c)

Fig. 2. Molecular models of 2 ring FF PNT in $\alpha$-helix conformation: (a) L-chiral isomer in $Y$-plane view, (b) L-chiral isomer in $Z$-plane view, (c) $\mathrm{D}$-isomer in $Y$ view, (d) $\mathrm{D}$-isomer in $Z$-plane view. Yellow cylinders represent the tube - coil of amino bonding [70].

Table 2. Lattice cell parameters for L-FF and D-FF PNT structures

\begin{tabular}{|l|l|l|}
\hline & $\mathrm{L}-F F^{a}$ & $\mathrm{D}^{a} \mathrm{FF}^{b}$ \\
\hline Temperature & $150 \mathrm{~K}$ & $140 \mathrm{~K}$ \\
\hline Space group & $\mathrm{P}_{1}$ & $\mathrm{P}_{5}$ \\
\hline$a, \AA$ & $24.0709(13)$ & $23.9468(14)$ \\
\hline$b, \AA$ & $24.0709(13)$ & $23.9468(14)$ \\
\hline$c, \AA$ & $5.4560(4)$ & $5.4411(2)$ \\
\hline$V, \AA^{3}$ & $2737.7(3)$ & $2702.2(2)$ \\
\hline $\mathrm{D}, \mathrm{g} / \mathrm{m}^{3}$ & 1.299 & 1.281 \\
\hline${ }^{a}[12],{ }^{b}[68,69]$ & \\
\hline
\end{tabular}

For a more detailed comparison and confirmation of differences for structures of different chirality, we also performed calculations for similar molecular structures based on experimental data in the "fixed position" or "single point" mode (Single Point - SP), that is, with fixed charges and distances between atoms. This construction was made as follows: we cut out similar ring molecular structures of FF (with the same 258 atoms) from the structure obtained from the experimental data of the crystallographic database of the Cambridge center of crystallographic data [69]. These data are denoted as CCDC 16337 for L-FF from [12] and 
CCDC 1853771 for D-FF from [68, 69]. The main parameters of these structures are presented in Table 2 and images are shown in Figure 3, where the selected FF rings are pointed out by yellow dashed circle lines.

The X-ray diffraction of single crystals allows distinguishing between the enantiomorphs $[105,106]$. Analysis performed in [68] revealed that the lattice cell parameters of D-FF PNT are close to those for L-FF structures reported by Gorbitz [12], but only their space groups are different (Table 2). As reported by Gorbitz, L-FF PNT structure belong to P61 space group, whereas the results obtained in [68] show that D-FF PNT must belong to P65 space group. Obviously, this symmetry difference is attributed to their monomer chirality. Although, the well-known 230 crystallographic space groups were deduced using a right-handed Cartesian coordinate system [107], a left-handed system is also frequently used in stereochemistry for the description of mirror-imaged enantiomers. This leads to 11 pairs of enantiomorphic space groups, including the P61-P65 pair [33], which is distinguished by the twisting direction of their 6-fold screw axis [107].

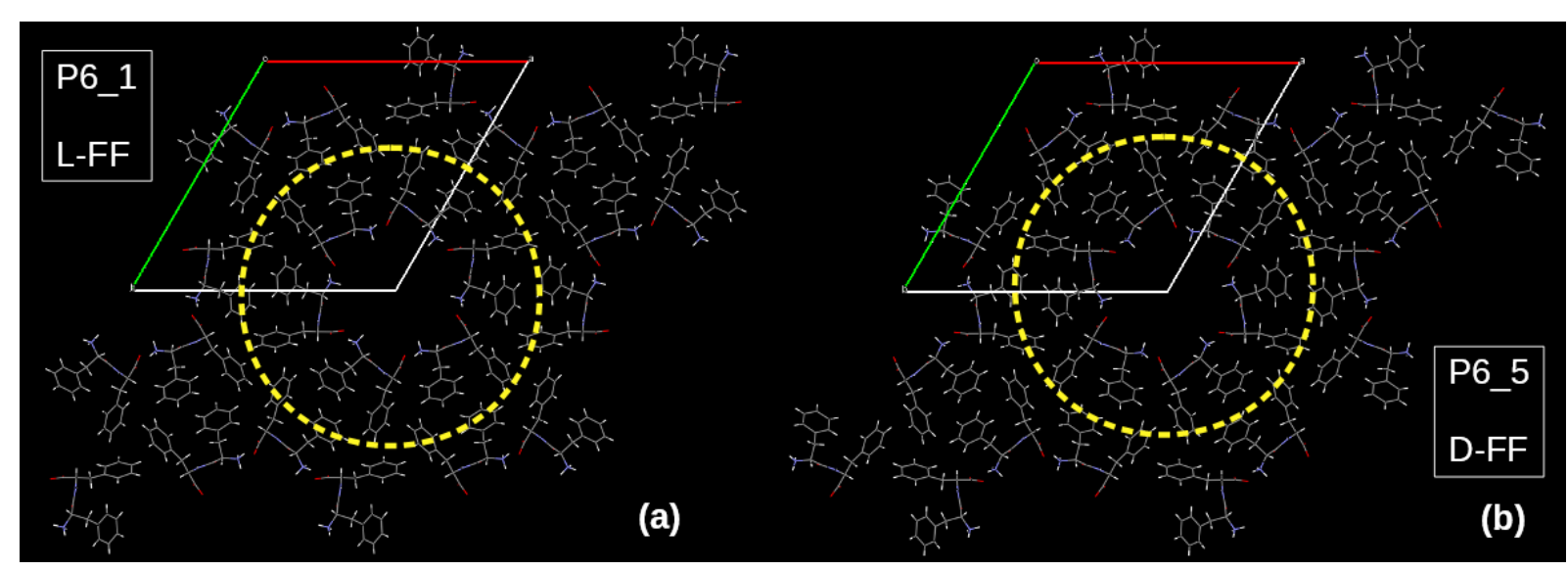

Fig. 3. Diagrams of the structural data based on the Cambridge Crystallographic Data Centre for two different FF enantiomers with symmetry elements and their general atomic positions for (a) L-FF with space group $\mathrm{P}_{1}$ and (b) D-FF with $\mathrm{P}_{5}$ space groups. The hexagonal unit cells are marked by green and red lines. The rings with atoms selected for calculations are shown by yellow dashed lines.

The selected FF rings with 258 atomic positions were transferred from Mercury *.cif format to the *.hin format in the HyperChem working space using standard procedure by OpenBabel program software [108]. After that similar atomic configurations of the FF rings in the HyperChem workspace were constructed and all necessary similar calculations were performed. The data obtained were analyzed, collected and now are presented in Figure 3 and in Table 3. Further, a similar procedure was performed for 2 rings FF models, for both L-FF and D-FF structures. The data obtained are shown in Table 4.

Both FF rings, obtained from experimental data, consist from 258 atoms, containing 6 dipeptides (di-phenylalanine FF from 43 atoms in the zwitter-ionic form, each $\mathrm{F}$ monomer is in the $\alpha$-helix conformation, not in a $\beta$-sheet, the only difference is in their chirality: L and D). The main difference between modelled and experimentally obtained structures is that all the modelled FF rings structures have an optimized geometry (using Polak-Ribiere conjugate gradient method from HyperChem package [71]), obtained during preliminary PM3 RHF/UHF calculations in [70], while FF rings structures based on experimental data are not optimized - they are extracted from samples existing in the crystal structure obtained during experimental studies and self-assembly [68].

It is important to compare these structures of the modelled structures and the taken-out rings with all their atomic positions as it were in experimental crystal structures - to determine their properties and relative changes. It is important to note that the latter 
experimental structures have their own "naturally-occurring" optimization in the process of self-organization and self-assembly of FF molecules into tubular structures under experimental conditions.
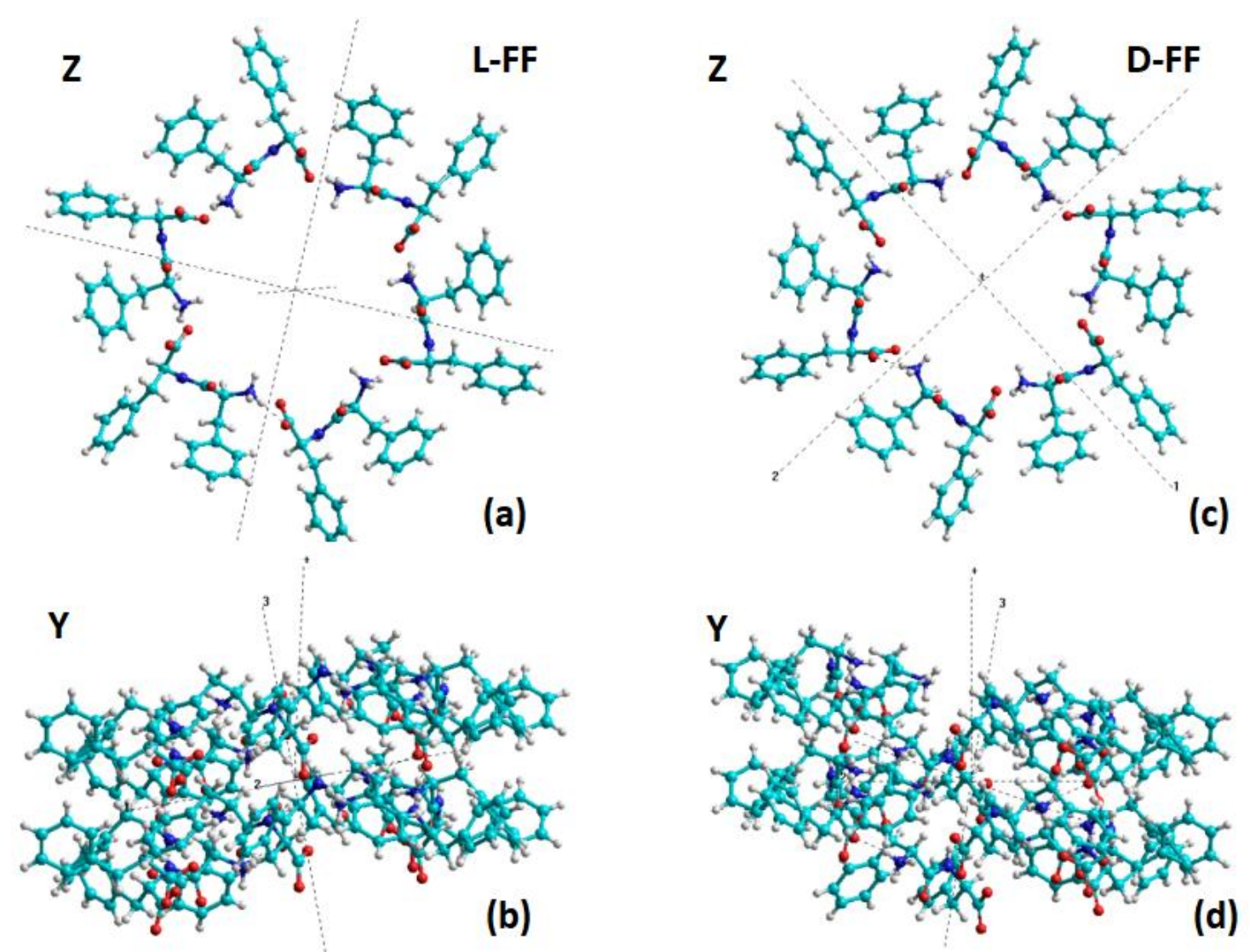

Fig. 4. Molecular models of a 2-ring PNT FF nanotube (FF PNT) in the $\alpha$-helix conformation, built on the basis of experimental crystallographic data: (a) L-chiral isomer in the Z-plane, (b) L-chiral isomer in the Y-plane, (c) D-chiral isomer in the Z-plane, (d) D-chiral isomer in the Y-plane.

However, the latter structures from experimental data have forms that are very different from modeled rings (see Tables 3 and 4). There are deep reasons for this important difference, and we are trying to study the origin of this difference. We will discuss this in greater detail below.

At first sight, the structures from the experimental data are not well-ordered rings; they have more randomly arranged atoms, as compared with more ordered structures which have more flat and regular ring shapes for the FF model structures.

It should be noted that these more "disordered" structures, obtained from experimentally optimized data, have higher total energy (and large RMS gradient of PES) and bad convergence during quantum PM3 calculations (both RHF and UHF). Note also that in this case we could not reach a final convergence of the total energy calculations for these L-FF and D-FF structures at their optimal distance point $c_{0} \sim 5.45 \AA$, equal to the lattice parameter along the $\mathrm{c}$ axis (as well as for other close distances in this neighborhood of the order of $\sim 10 \AA$ ). But for this case, we used comparable and similar other semi-empirical methods AM1 [71, 99], which helped us to calculate the total energies of the systems under study at these points with the necessary convergence and accuracy. As is known, PM3 is a reparameterization of AM1 [99], PM3 differs from AM1 only in the values of the parameters. Typically, non-bonded interactions are less repulsive in PM3 than in AM1. 
BYSTROV et al.

Table 3. Basic physical data for simulated and experimental FF structures

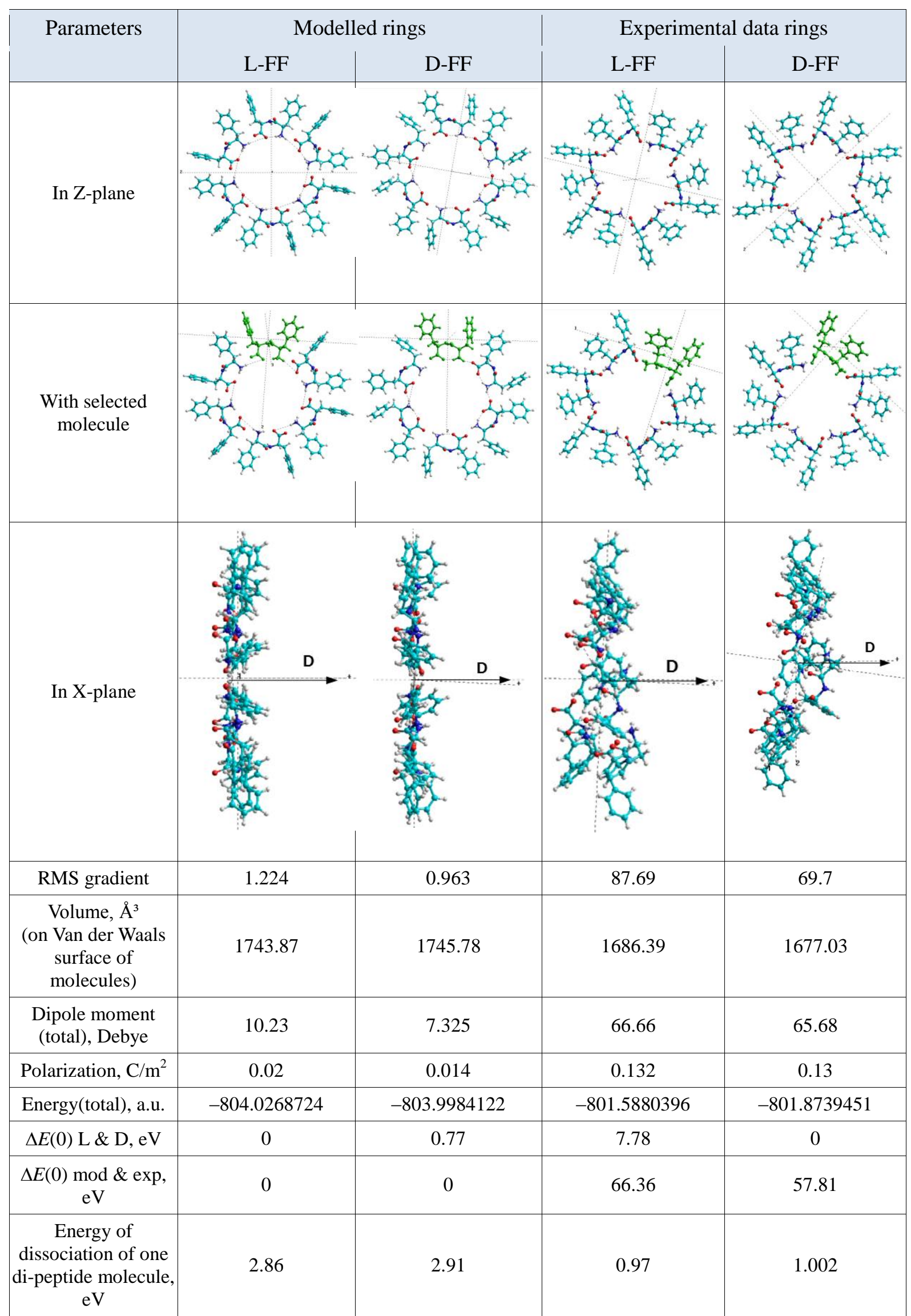

To compare these AM1 data with the PM3 calculations we used the Lennard-Jones type potential as a mathematically simple model that approximates the interaction of a pair of 
neutral molecules [109]. For more complex cases, the generic (generalize) form of the L-J function is often used. In this paper we use the following approximation:

$$
V_{g L J}(r)=C_{m n} V_{0}\left[\left(r_{0} / r\right)^{m}-2\left(r_{0} / r\right)^{n}\right],
$$

where $V_{0}$ is the depth of the potential well, $r$ is the distance between the molecules, and $r_{0}$ is the distance at which the potential reaches its minimum. At $r_{0}$, the potential function has the value $-V_{0}$.

Here the coefficient $C_{m n}$, is chosen so that the minimum value $V(r)=-V_{0} ; m$ and $n$ are variable coefficients describing the deviation of the behavior of relation (1) from the form of the behavior of the standard L-J potential, in which $m=12, n=6$. In the case of our calculations, their values are determined during the corresponding fitting (approximation).

In usual standard form of L-J function the $r^{-12}$ term, which is the repulsive term, describes Pauli repulsion at short ranges due to overlapping electron orbitals, and the $r^{-6}$ term, which is the attractive long-range term, describes attraction at long ranges (van der Waals force, or dispersion force). For more complex cases, the generalized form of L-J function (1) is often used.

\section{THE SIMULATION RESULTS AND STUDIES OF THE INTERACTION OF THE RING STRUCTURES OF FF PNT NANOTUBES ALONG THEIR MAIN AXIS}

To determine the nature of the interaction of individual ring structures during selfassembly and the formation of FF PNT nanotubes, we placed them at a relatively large distance from each other (of the order of $r \sim 100 \AA$, at this distance the relative changes in the total energy are already almost within the calculation error) along the main c-axis nanotubes, and then gradually brought them together and carried out the calculation of the total energy at each step. Thus, the total energy profile $E(r)$ and the position of the energy minimum $E_{0}\left(r_{0}\right)$ at $r=r_{0}$ were determined, when the optimal configuration of the nanotube was achieved. The calculations were carried out by different methods. Let us consider the results of quantum chemical calculations using the PM3 and AM1 methods (in RHF, since UHF gives the same energies here). Figure 5 shows an example of such a PM3 calculation for a model of 2 L-FF PNT rings.

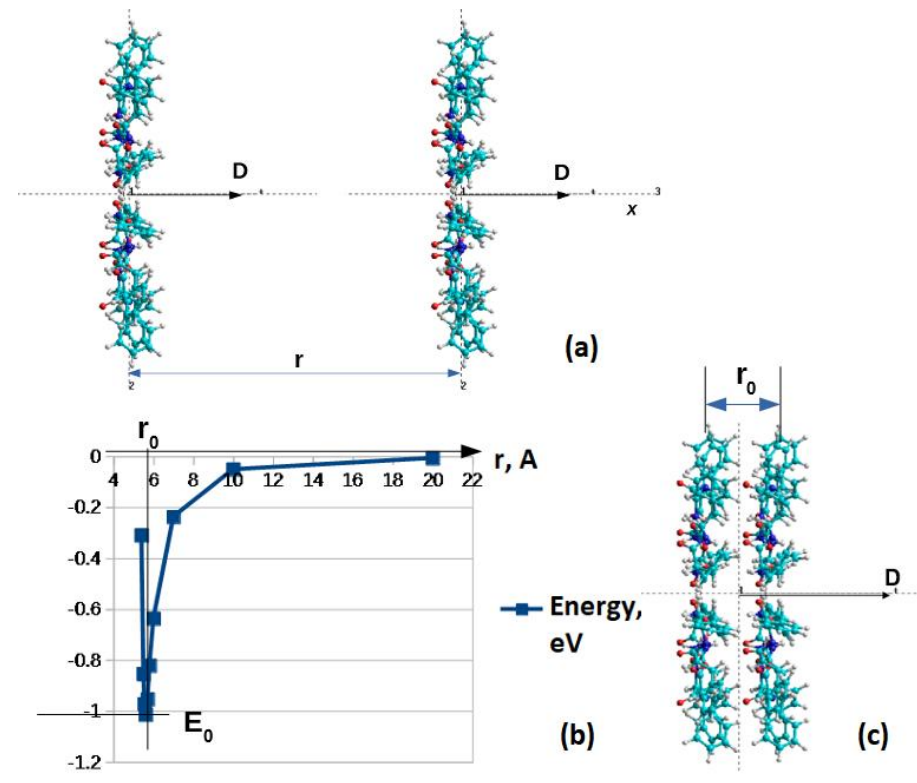

Fig. 5. Total energy profile from the distance for 2 interacting rings of the L-FF PNT model using the PM3 RHF method: a) location of rings at a distance $r, \mathbf{b})$ total energy profile with optimal parameters $E_{0}$ $\left.\left(r_{0}=5.6 \AA\right)=-1.014 \mathrm{eV}, \mathbf{c}\right)$ arrangement of rings at $r=r_{0}$. The arrows indicate the direction of the dipole moment D. 
Similar calculations were carried out for the cases with rings of the D-FF PNT model, as well as with ring structures isolated and obtained from their experimental data. It should be noted that the optimization of the model structures carried out by us turns out to be rather a complicated process and is not always unambiguous. This is determined by the complex structure of PES - the mathematical surface of the total energy, each point of which depends on the location of all the atoms of the system and which, as a rule, has more than one local minimum and the gradient descent to the absolute minimum can occur in different ways. At the same time, it still depends on the initial position - from different initial configurations one can arrive at different final states. As an example, we optimized the rings of the D-FF PNT model with a different initial configuration and arrived at a more symmetrical form of the DFF rings. However, the total energy in the minimum turned out to be higher (below, in Fig. 6 of this model, the D-FF model 2 corresponds to the blue graph). Apparently here this is precisely the case of hitting a local minimum with a higher energy. Another difficulty arose in the calculation of ring structures based on the experiment: at close distances, the calculation convergence is violated (Fig. 6).

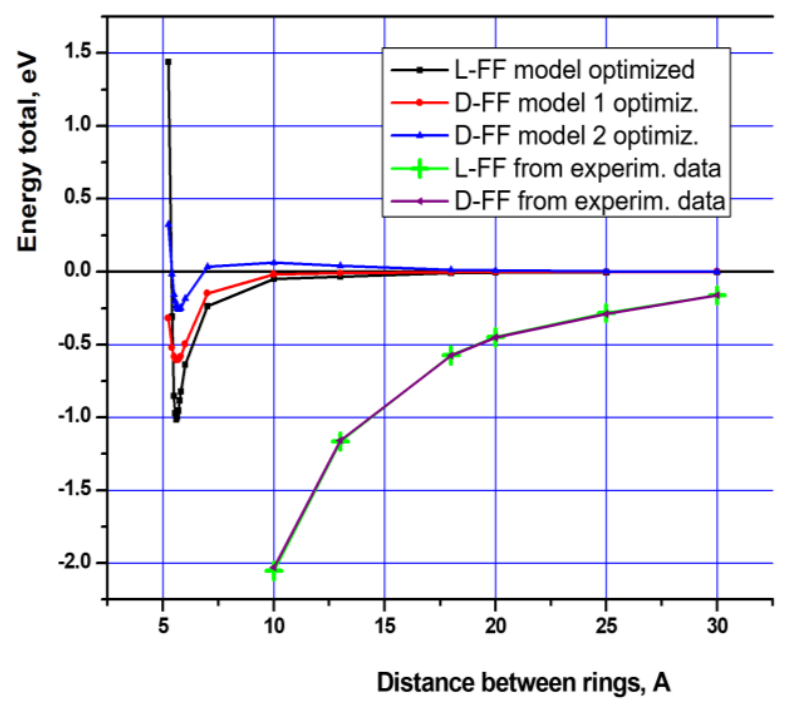

Fig. 6. Energy profiles calculated by the PM3 RHF quantum method for both model optimized PNT rings of different chirality L-FF, D-FF, and ring structures extracted from experimental crystallographic data (data are shown up to $10 \AA$, closer there is no convergence of PM3 calculation algorithm).

Therefore, we proceeded to calculations by another AM1 RHF quantum chemical semiempirical method, in which the convergence of the calculation algorithm was performed at all distances, and to approximation of the calculation results by the method of generalized potential L-J. Similarly, calculations were performed for structures with D-FF chirality.

To compare these calculations using the AM1 and PM3 methods (in the RHF approximation), we calculated the total interaction energy of two "ring" structures, L-FF and D-FF, with the distances between them. Then fittings (approximations) were performed with the generalized L-J function (1) for both the cases - AM1 and PM3 (both for the case of L-FF and for D-FF). The data of quantum calculations by the AM1 method gave us good convergence at any distances for all cases.

We compared the fitting data (approximations) for calculations using the AM1 and PM3 methods, and as a result we obtained the $V_{0}\left(r_{0}\right)$ values for PM3 (for the cases of L-FF and DFF). The latter data are presented in Figure 7 and in Tables 4. 


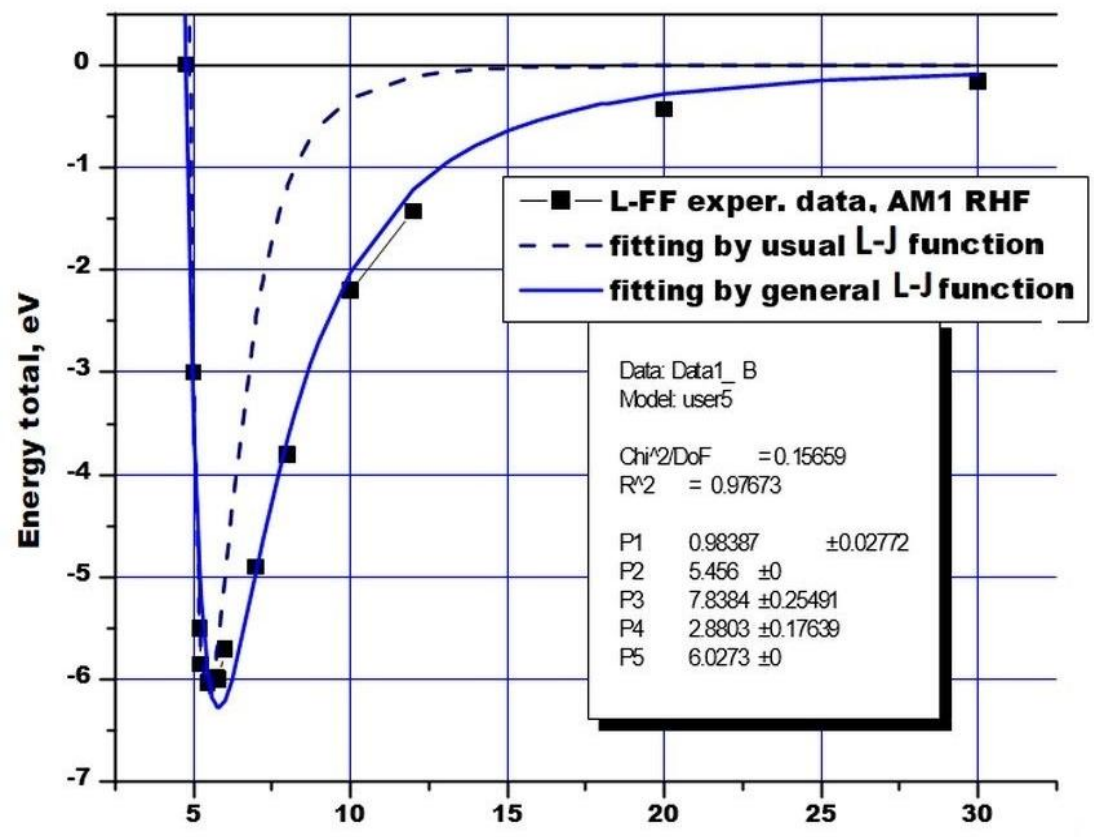

Distance between rings, $\AA$

a)

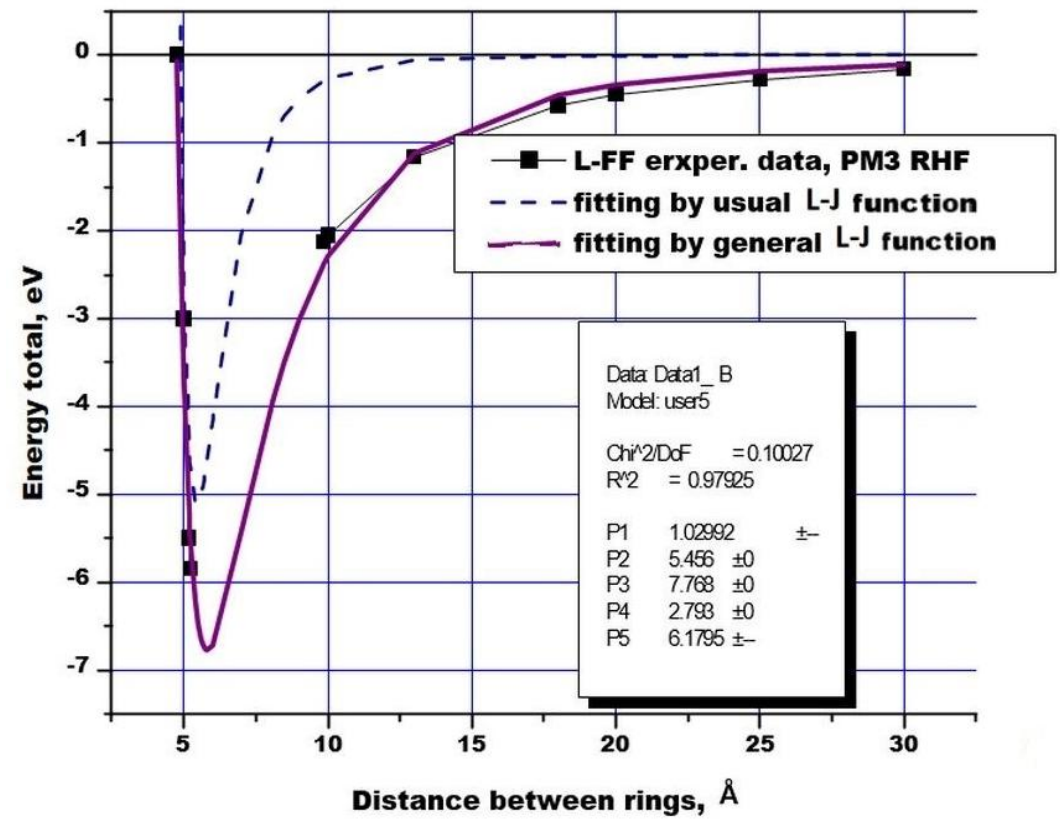

b)

Fig. 7. Results of the calculation of energy profile for 2-ring-shaped L-FF PNT structures (according to the experiment): a) using the AM1 RHF method and approximation of this profile using the generalized function L-J (1), b) using the PM3 RHF method (partially, according to the convergence region calculation) and approximation of energy profile based on the parameters of the generalized function L-J (1), obtained by fitting AM1. Here the fitting parameters and the parameters in the formula (1) are designated as: $C_{m n}=\mathrm{P} 1, r_{0}=\mathrm{P} 2, m=\mathrm{P} 3, n=\mathrm{P} 4, V_{0}=\mathrm{P} 5$.

The values of the coefficients determined after fitting are the following (see Fig. 7):

D-FF: $\quad C_{m n}=0.9569 \sim 1, \quad m=6.402 \sim 6.4, \quad n=2.793 \sim 2.8, \quad$ for $r_{0}=5.441 \AA$ and $V_{0}=6.21998 \sim 6.22 \mathrm{eV}$

L-FF: $\quad C_{m n}=1.0299 \sim 1, \quad m=7.768 \sim 7.8, \quad n=2.793 \sim 2.8, \quad$ for $\quad r_{0}=5.456 \AA \quad$ and $V_{0}=6.1795 \sim 6.18 \mathrm{eV}$. 
The values of the obtained coefficients $m$ and $n$ strongly deviate from the standard values ( $m=12, n=6$ ) in the L-J relation (1), obviously, due to the strong influence of the quantum interactions of the electronic subsystems in these cases of complete quantum calculations.

It should be noted that the value of the energy in the minimum (optimum) for these structures of different chirality is not greatly different here and the energy varies only slightly with the distance between the interacting clusters.

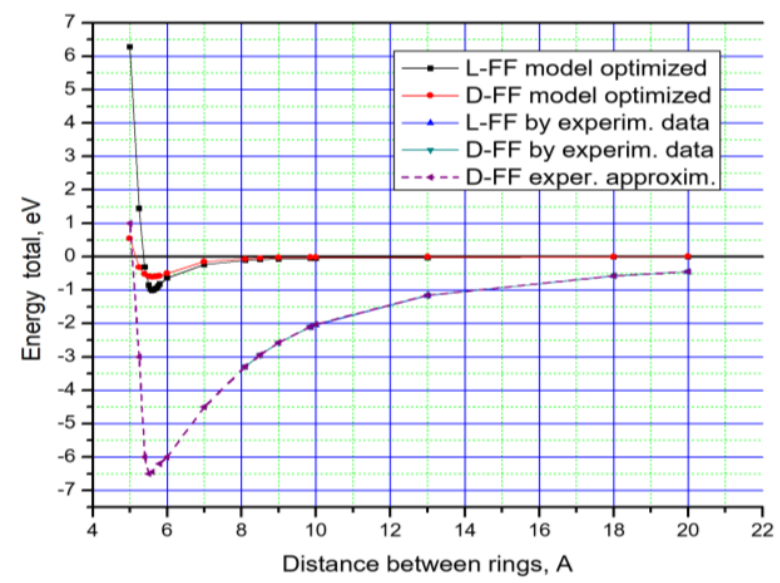

Fig. 8. The energy profiles of both types of structures in their different chirality according to the results of quantum chemical calculations.

Table 4. Structures and physical properties calculated for the model and experimental structures FF with two rings and coils of the spiral - calculated by the method of PM3 RHF

\begin{tabular}{|c|c|c|c|c|}
\hline \multirow[t]{2}{*}{ Parameters } & \multicolumn{2}{|c|}{ Modelled rings } & \multicolumn{2}{|c|}{$\begin{array}{c}\text { Experimental data of ring-like } \\
\text { structures }\end{array}$} \\
\hline & L-FF & D-FF & L-FF & D-FF \\
\hline In $X$-plane & $8 v+\frac{2 \pi}{50}$ & $x \quad \frac{x}{4}$ & & \\
\hline $\begin{array}{l}\text { Dipole moment, } \\
\text { Debye }\end{array}$ & 23.812 & 16.330 & 139.7 & 137.7 \\
\hline Polarization, $\mathrm{C} / \mathrm{m}^{2}$ & 0.023 & 0.0156 & 0.13845 & 0.13725 \\
\hline $\begin{array}{l}E_{0} \text { Energy(total), a.u. } \\
\text { at optimal distance } z_{0}\end{array}$ & -1608.090321475 & -1608.019186 & -1603.4033553216 & -1603.97716 \\
\hline $\begin{array}{l}\Delta E_{0}=\Delta E\left(x_{0} / x_{m}\right)=V_{0} \\
\mathrm{eV}\end{array}$ & -1.014 & -0.607 & -6.18 & -6.22 \\
\hline $\begin{array}{l}\text { Distance between } \\
\text { rings at optimal } x_{0}, \AA\end{array}$ & $\sim 5.56-\sim 5.6$ & $\sim 5.42-\sim 5.6$ & 5.456 & 5.441 \\
\hline
\end{tabular}

Moreover, as can be seen, the energy values at the minimum (optimum) for the structures, based on the data from the experiment, turn out to be noticeably lower (more than $\sim 6 \mathrm{eV}$ ) as compared to our model structures (of the order of $\sim 1 \mathrm{eV}$ ). These results are presented in 
Figure 8. At the same time, the values of the dipole moment and polarization for them turn out to be significantly higher than for the model structures. These data are summarized and shown below in Table 4.

Such behavior of ring-shaped structures, constructed from their experimental crystallographic data, speaks of their better natural optimization in the process of selfassembly under experimental conditions, compared with our model ring structures. And this is due to the fact that here the structures are no longer rings, but coils of a helix. Therefore all the self-organized structures in the process of self-assembly have the form of helices formed inside the crystals (see below Fig. 9). Moreover, the screw (rotation) of these helices has the character of alternation with respect to the initial chirality of amino acids: L-FF forms the right screw, and D-FF - the left screw of the helix. This is exactly the same as the established pattern in [28-30].

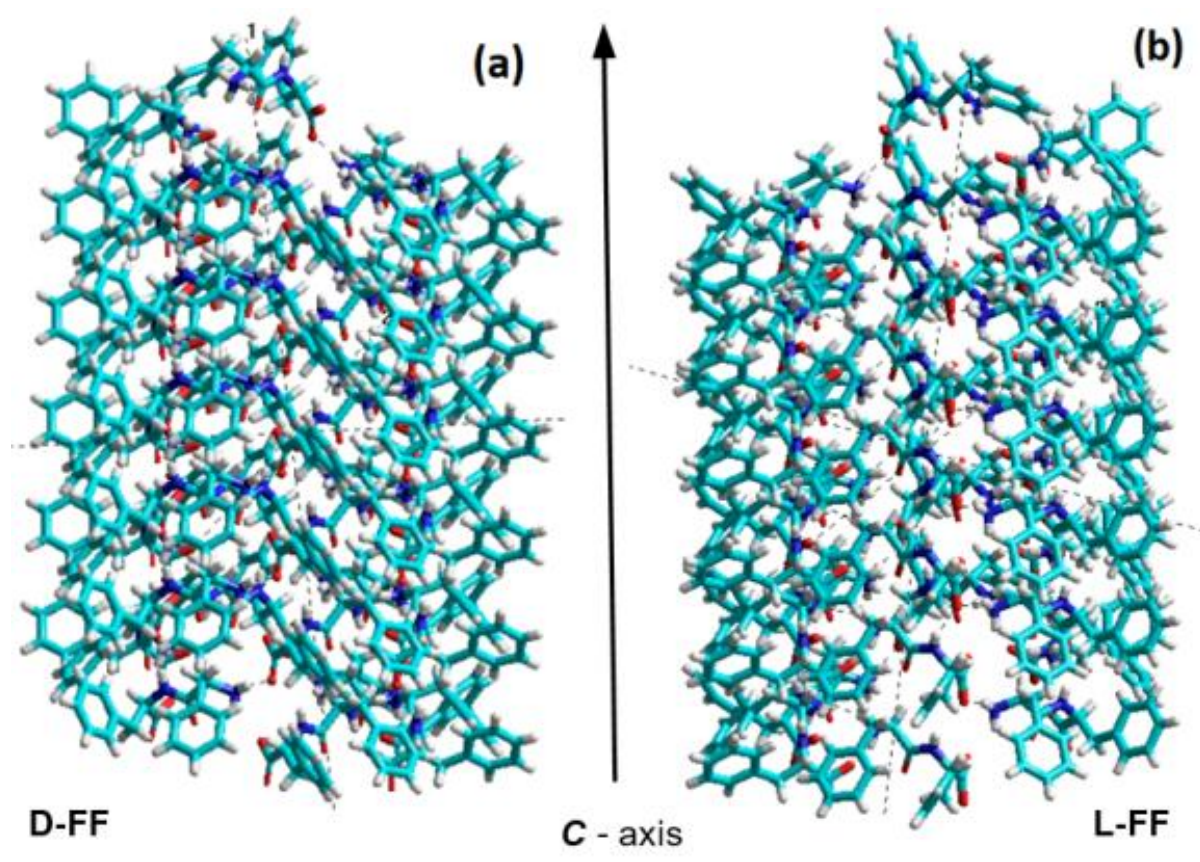

Fig. 9. Various helix formations in experimental crystal structures of FF PNT for different chiralities of the initial molecules: D-FF (a) and L-FF (b). Different PNT twists occur on the basis of L-FF and D-FF: the right (D-chiral) $\alpha$-helix for L-FF and the left (L-chiral) $\alpha$-helix for D-FF.

Thus, it can be concluded that these ring-shaped structures cut from the experimental data of the crystal structure have a different atomic order here. They represent a part (loop) of a helix or annular helical structure with a step that is directly equal to the cell parameter with $c \sim 5.4 \AA$ along the main $c$-axis of the nanotube. And this axis passes through all the crystal structures along the $\mathrm{c}$ axis and forms spiral structures that differ between $\mathrm{L}$ and $\mathrm{D}$ enantiomeric forms by means of a rotation screw: from the initial L-FF form of amino acids with L chirality, forms a chiral D-helix with right-handed rotation, while from the initial structure of the amino acids D-FF with chirality D, a L-chiral helix with a left-handed rotation arises (Fig. 9).

Consequently, it is neither a chaotic nor a poorly ordered structure, but another topological structure - it is not a ring, but a helix coil. And all the atoms in it not randomly distributed, but have their own order along this loop of the helix in the course of one rotational step along the $c$-axis. Important that these structures, both L-FF and D-FF, were obtained automatically during self-assembling process. This means that these structures are naturally-occurring optimized. And these naturally optimized helix structures have the lowest total energy as compared to simulated optimized ring structures (see Fig. 8 and Table 4). 
This result is fully consistent with the rules established recently by Tverdislov's group [28-30, 48-50]. This means that different twisting of the L-FF and D-FF PNT leads to a change in their sequence of chirality when the structural organization is complicated: the formation of the right (D-chiral) $\alpha$-helix changes the chirality of the original left amino acids L-FF, while left-side (L-chiral) $\alpha$-helix is observed for the original right-side amino acids DFF. These are important general rules.

In order to study this phenomenon more deeply, to check and confirm these rules, further we carry out a number of new calculations of the energy of these interacting various chiral FF structures along directions of different crystallographic axes.

As a result, we can evaluate the priority directions of formation and growth of nanostructures based on dipeptides (diphenylalanine) from amino acids of different chirality and thus, determine the main dynamics of self-assembly and get some model that allows us to explore this process of self-assembly and its key features.

\section{RESULTS OF MODELING AND STUDYING THE INTERACTION OF THE RING STRUCTURES OF FF PNT NANOTUBES IN THE VARIOUS DIRECTIONS}

For this purpose, more detailed calculations of the total energy profiles for both L-FF and D-FF structures, both for simulated and experimental ones, were carried out. Based on the quantum calculations obtained above, we now use here faster molecular-mechanical (MM) methods for detailed calculations of these energy profiles (see below). The main changes of the energy are studied along the main axis of the interacting FF rings with two different orientations: 1) along the main tube and crystal $c$-axis and 2) in the perpendicular direction along $a$-axis of the crystal structure.

For all the cases, the simulated L-FF and D-FF structures were optimized using the PM3 quantum-chemical semi-empirical method (mainly in the RHF approximation, since UHF showed basically the same values), and then the calculations were carried out using molecular-mechanical methods, such as $\mathrm{MM}+$, BIO CHARM and Amber taking into account the effective fixed charges already received. These approaches allow us to calculate the data faster at each step by changing the distance, while quantum calculations for these cases need very long time. The absolute values of the energies in these cases, of course, will differ, but for our purposes just the relative changes between different structures are important. Of significance is the relative energies changes over long distances become very small (in the limits of calculation error), and then they can be neglected (this is approximately at a distance of 50-100 $\AA$ between the centres of the molecular clusters).

Note that the main relative changes in the total energy with respect to the distances and geometry of the systems (the depth and position of their minimum at optimum distances) are approximately at the same distances for these different approaches.

The changes in the total interaction energy of the rings of the FF model structures (as well as the helix coils or coil extracted from the experimental crystal structures) depending on the distances between them are presented in the graphs in Figure 10 (for the case of only one moving ring FF and distances between two rings FF, we deal with changes along the $c$-axis and along the $a$-axis). In Figure 11 the double FF pairs move relative to one another. It is shown only for the case of changing the distance along the $c$-axis.

At the same time, for simplicity of illustration, these figures show only the model rings, while the graphs show all the cases of all investigated structures, including those obtained from experimental data.

The Figures 10 and 11 show the results for the MM+ method only; other used methods give the similar results. All the data obtained for the positions of the optimal distances with the minimum energy of the systems at these points relative to the maximum distances (they were chosen $\sim 100 \AA$, when the energy changes become comparable with the calculation error), are collected in Table 5. 
To understand better the physics of the interaction processes of the studied molecular ring structures when they self-assembly with tubular structures, we made an approximation (fitting) of the calculated total energy profile between the two interacting structural units using MM methods (rings for modeled structures and an annular spiral coil for experimental structures) and calculated this fitting using the generalized Lenard-Jones formulas L-J (1), just as for the quantum calculations above. The results of this fitting are shown in Figure 12.
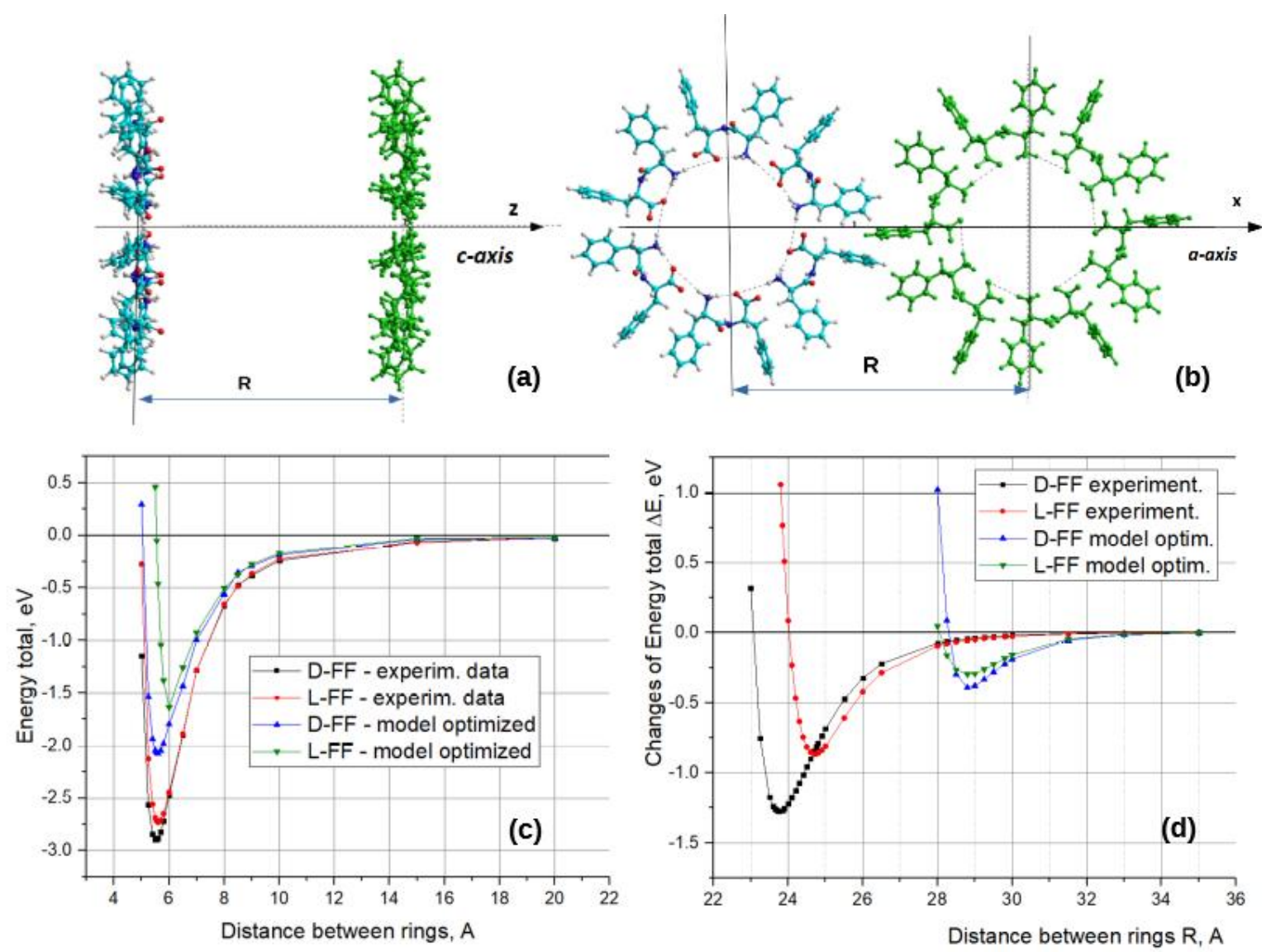

Fig. 10. The calculated changes in the total energy when the distance between two different rings FF changes: (a) and (b) are the two main mutual configurations of the rings FF and the directions of the distances between them; (c) and (d) - calculated energy profiles depending on the distance between the FF rings; the results are calculated using the MM + method (after optimization and the PM3 RHF quantum method for simulated structures), in comparison with the data for experimentally optimized similar structures in the form of coils. For simplicity, the upper images (a) and (b) of floating rings are shown only for the case of L-FF structures in their simulation. All other cases are similar.

But in these cases, using the MM + method, the values of the coefficients $m$ and $n$, obtained from equation (2), turned out to be much closer to the standard values in the ratio L-J (1) and have the following data (see Fig. 12):

D-FF: $\quad C_{m n}=0.99926 \sim 1, \quad m=11.6114 \sim 11.6, \quad n=5.636 \sim 5.6, \quad$ for $r_{0}=5.5 \AA \quad$ and $V_{0}=2.8939 \sim 2.89 \mathrm{eV}$

L-FF: $\quad C_{m n}=0.99931 \sim 1, \quad m=11.6255 \sim 11.6, \quad n=5.7437 \sim 5.7$, for $r_{0}=5.6 \AA$ and $V_{0}=2.7301 \sim 2.73 \mathrm{eV}$.

The obtained result means that in both the cases (for calculations with fixed charges and without direct influences of quantum effects of electronic subsystems on each calculated step of changing the distance) the interaction between these two molecular structures occurs close to the standard L-J ratio. Therefore, the interaction between these two molecular clusters corresponds to the general electrostatic coulomb law for complex molecular systems that have a distribution of charges and dipoles in their structures. This is completely similar to the 
situation described in $[55,56]$ for two interacting large molecular structures, including systems with different chiralities.
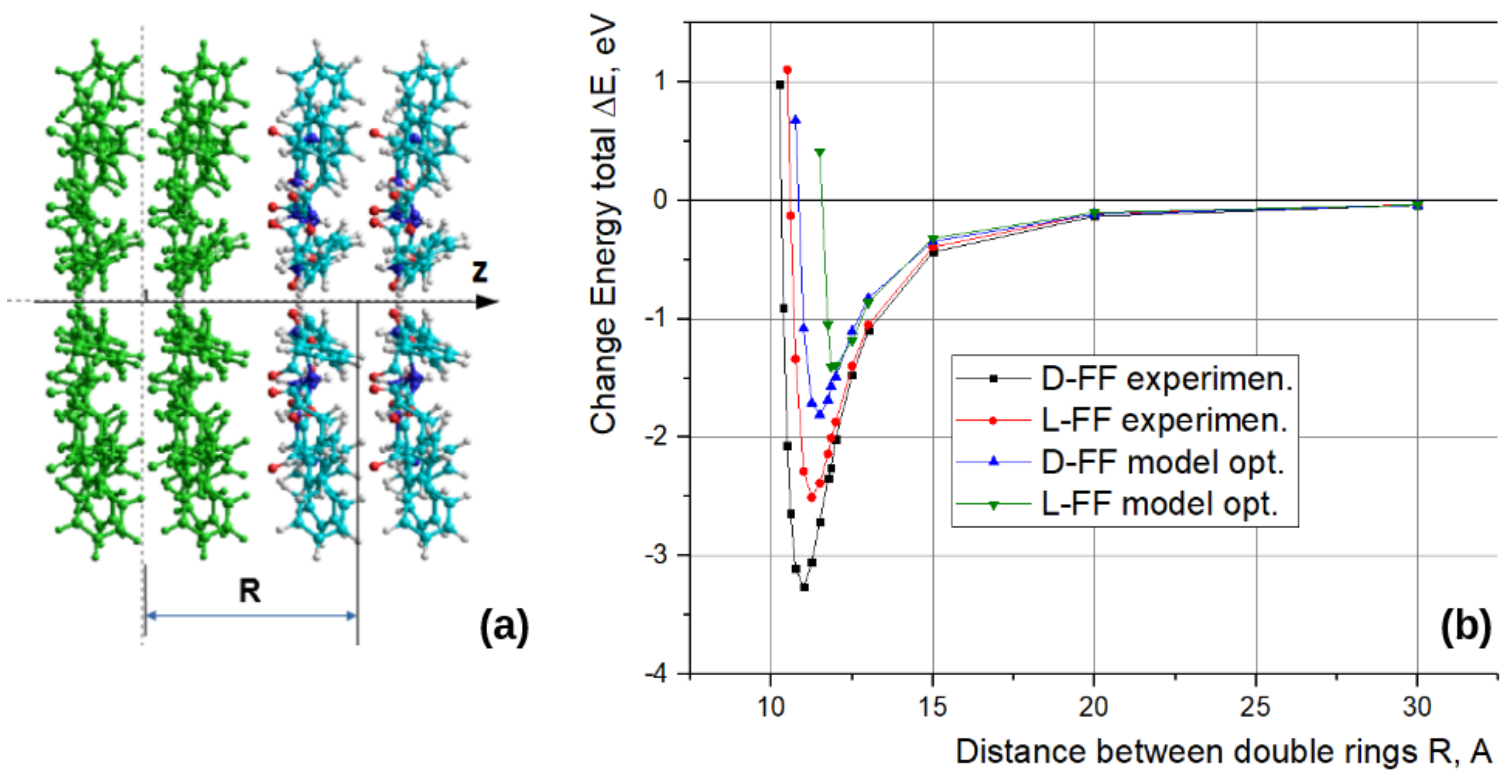

Fig. 11. The calculated total energy profiles for the case of movable double rings FF: (a) the configuration of the system of 2 double rings FF at the optimal distances between them; (b) the calculated energy profiles by the MM + method for various model and experimental FF structures under the same conditions as for the motion of a single FF ring. The optimal distance for double FF pairs is twice as large as for single FF rings, but the minimum energies are about the same.

For large distances $r$, molecules attract, which corresponds to the term $r^{-6}$ in the formula L-J (1). This dependence can be substantiated theoretically, and takes place due to the Van der Waals forces (dipole-dipole induced interaction). For small distances $r$, however, the molecules repel because of the exchange interaction (when the electron clouds overlap, the molecules begin to repel strongly), to which the term $r^{-12}$ corresponds in (1).

In our case, in the approximation of molecular mechanics approaches, we obtained that by approximating the interaction of ring-like molecular structures using the generalized formula (1), we obtained slightly different values of parameters in the ratio L-J (1): $m \sim 11.6$, which is close to $m=12$, and $n \sim 5.6$ for $\mathrm{D}-\mathrm{FF}$ ( $~ 5.7$ for L-FF), which is also close to $n=6$. That is, the interaction of these structures in the process of self-assembly into a tubular structure corresponds to the interaction physics of large electrostatic and dipole (and more generally multipole) molecular clusters. 


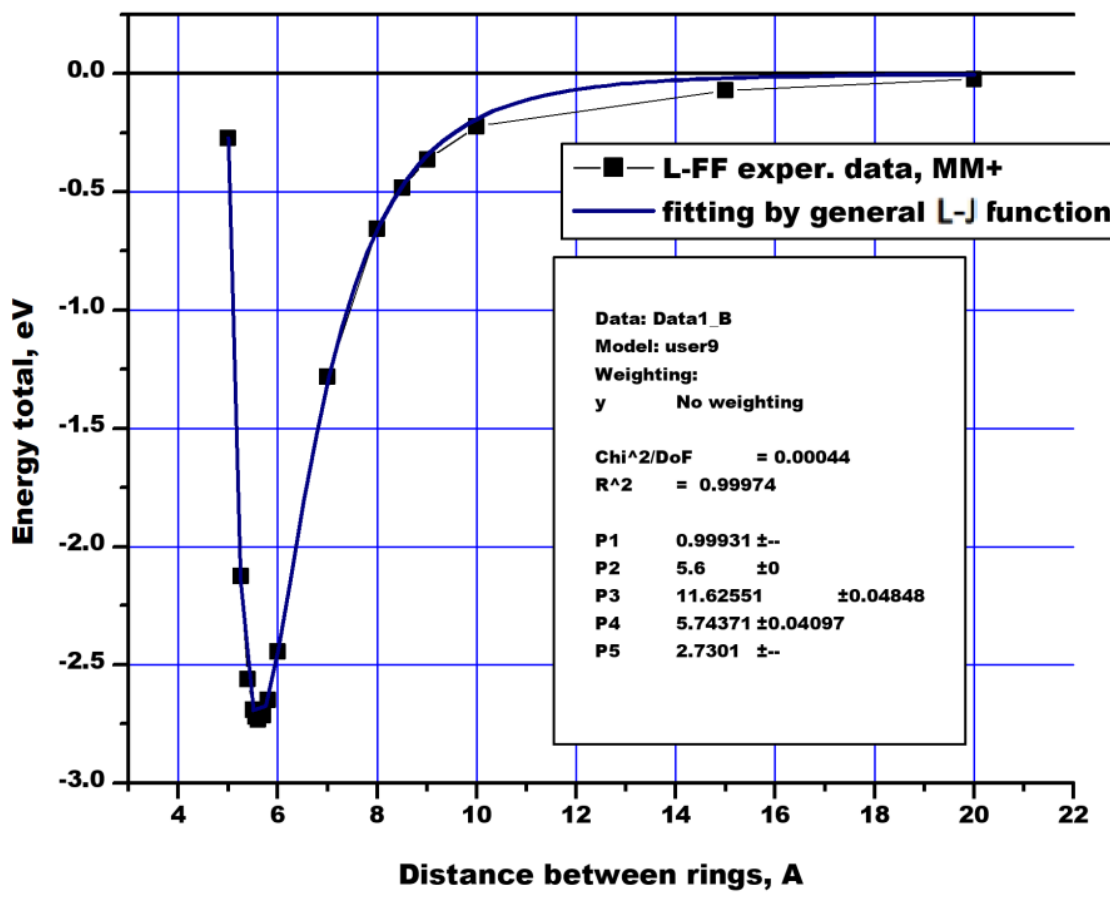

(a)

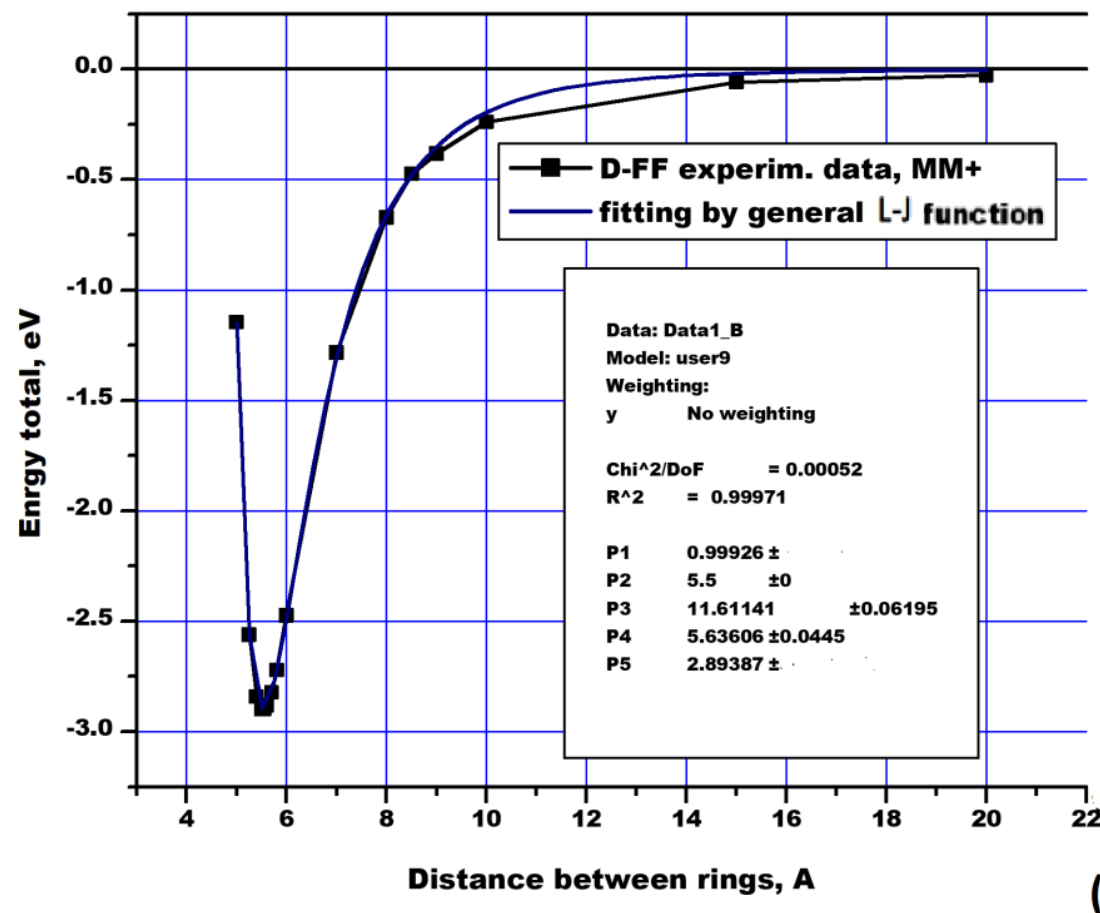

Fig. 12. The results of the approximation (fitting) for the molecular-mechanical method MM + calculation (after the quantum semi-empirical calculation of PM3 RHF) based on ring-shaped units of the "spiral turn" obtained from experimental data: a) for chirality L-FF (parameters P3 $=m=11.6255 \sim 12$ were obtained, and $\mathrm{P} 4=n=5.7437 \sim 6$, which are close to the usual standard of the L-J potential; b) for chirality D-FF (the parameters $\mathrm{P} 3=m=11.6114 \sim 12$ and $\mathrm{P} 4=n=5.636 \sim 6$ are obtained, also close to the usual standard ratio in the L-J potential. 
BYSTROV et al.

Table 5. Equilibrium parameters for interacting rings of different enantiomers of FF

\begin{tabular}{|c|c|c|c|c|c|c|c|c|c|c|}
\hline & \multicolumn{4}{|c|}{ Model ring } & \multirow{3}{*}{$E_{0}(\mathrm{D}) / E_{0}(\mathrm{~L})$} & \multicolumn{4}{|c|}{ Experimental spiral turn (coil) } & \multirow{3}{*}{$E_{0}(\mathrm{D}) / E_{0}(\mathrm{~L})$} \\
\hline & \multicolumn{2}{|c|}{ L-FF } & \multicolumn{2}{|c|}{$\mathrm{D}-\mathrm{FF}$} & & \multicolumn{2}{|c|}{ L-FF } & \multicolumn{2}{|c|}{ D-FF } & \\
\hline & $E_{0}, \mathrm{eV}$ & $r_{0}, \AA$ & $E_{0}, \mathrm{eV}$ & $\mathrm{r}_{0}, \AA$ & & $E_{0}, \mathrm{eV}$ & $r_{0}, \AA$ & $E_{0}, \mathrm{eV}$ & $r_{0}, \AA$ & \\
\hline \multicolumn{11}{|c|}{ Single } \\
\hline \multicolumn{11}{|c|}{$\operatorname{Dir} c$} \\
\hline MM+ & -1.759 & 5.9 & -2.076 & 5.6 & 1.18 & -2.732 & 5.5 & -2.896 & 5.5 & 1.06 \\
\hline $\begin{array}{l}\text { BIO } \\
\text { CHARM }\end{array}$ & -1.846 & 6.0 & -1.943 & 5.6 & 1.05 & -3.746 & 6.0 & -6.446 & 5.55 & 1.7 \\
\hline Amber & & & & & & -6.313 & 5.6 & -6.739 & 5.5 & 1.07 \\
\hline Average & & & & & 1.115 & & & & & 1.27 \\
\hline \multicolumn{11}{|c|}{ Dir $a$} \\
\hline $\mathrm{MM+}$ & -0.139 & 28.8 & -0.095 & 29.8 & 0.683 & -0.327 & 24.8 & -0.414 & 23.8 & 1.3 \\
\hline $\begin{array}{l}\text { BIO } \\
\text { CHARM }\end{array}$ & -0.157 & 28.6 & -0.071 & 29.2 & 0.449 & -0.129 & 25.0 & -0.398 & 23.8 & 3.08 \\
\hline Amber & -0.142 & 28.6 & -0.052 & 30.0 & 0.362 & -0.357 & 24.8 & -0.410 & 23.8 & 1.2 \\
\hline $\begin{array}{l}\text { Amber } \\
\varepsilon \text { const }\end{array}$ & & & & & & -0.234 & 24.8 & -0.393 & 23.8 & 1.7 \\
\hline Average & & & & & 0.498 & & & & & 1.82 \\
\hline \multicolumn{11}{|c|}{ Double } \\
\hline \multicolumn{11}{|c|}{$\operatorname{Dir} c$} \\
\hline MM+ & -1.40 & 5.93 & -1.81 & 5.75 & 1.29 & -2.508 & $\begin{array}{c}11.24 / 2 \\
5.62\end{array}$ & -3.265 & $\begin{array}{c}11.0 / 2 \\
5.5\end{array}$ & 1.3 \\
\hline $\begin{array}{l}\text { BIO } \\
\text { CHARM }\end{array}$ & -1.457 & 6.0 & -1.576 & 5.75 & 1.08 & -* & $\begin{array}{c}12 / 2 \\
6.0\end{array}$ & -3.233 & $\begin{array}{c}11 / 2 \\
5.5\end{array}$ & - \\
\hline \multicolumn{11}{|c|}{ Dir $a$} \\
\hline MM+ & -0.295 & 28.8 & -0.392 & 28.8 & 1.32 & -0.863 & 24.7 & -1.273 & 23.75 & 1.5 \\
\hline $\begin{array}{l}\text { BIO } \\
\text { CHARM }\end{array}$ & -0.32 & 29.8 & -0.42 & 29.8 & 1.31 & -* & 24.8 & -1.230 & 23.8 & - \\
\hline
\end{tabular}

* there is no result of calculating this unstable state by this method - the minimum energy cannot be obtained, but there is only an inflection point on the curve (a function of our graphs or profile change) of energy at the optimal distance.

\section{MAIN RESULTS AND DISCUSSION}

\subsection{Condensation energies and the effect of chirality}

One of the main conclusions suggested by these computed data is concerned with the interaction energies between FF units, which could be considered as condensation energies $E_{0}$ for the self-assembly process of the FF PNT formation.

First, it turned out that this condensation energy along the main $c$-axis of the tubes is approximately close between L and D structures, while the condensation energy in the perpendicular direction - along the $a$-axis, has more differences between the structures of different chirality D and L: for D-FF structures, the change in energy at its minimum has larger values than for L-FF. That is, in this direction, the condensation energy of D-FF structures is larger in absolute value than for L-FF structures. And at the same time, the 
optimal distances were also closer (see Fig. 10,d and Table 5). It also means deeper and denser condensation and layout of the D-FF PNT units during the self-assembly process, compared to the L-FF PNT. As a result, the D-FF PNT is formed as a thicker, wider and shorter cylindrical (or tubular) sample than the L-FF PNT. It is important that this effect is already observed experimentally [68], which confirmed our approaches and simulation results.

Obviously, this effect is physically due to the initial difference in the magnitude and orientation of the dipole moments of the D-FF and L-FF structures (Fig. 1, Table 1), which leads to a slightly different dynamics of the electrostatic interaction of both the dipoles of single FF molecules (L-FF and D-FF) and the forming ring structures, their subsequent packaging into tubular structures and the interaction of individual nanotubes during their selfassembly into a bundle of such nanotubes - as is observed in experiment [68]. These experimentally obtained data [68] showed that D-FF nanotubes are thicker and shorter than LFF. Why is this possible? If we look at the condensation energy data calculated by us (see Table 5), we find that:

1) the condensation energy along the nanotube along the c axis between two rings is about 2-3 eV for both L-FF and D-FF PNT (a deeper decrease for experimental cases), this is comparable with previous calculations for the FN PNT in $[18,19]$ (which were found to be approximately $4-5 \mathrm{eV}$ ) but in this case it was believed that PNT is self-organizing from FF in the conformation of the $\beta$-sheet, which could have a stronger interaction along the main axis of the nanotube between the FF $\beta$-rings leaf relative to the FF $\alpha$-rings;

2 ) the condensation energy in the perpendicular direction to the main axis of the nanotubes along the a axis is about $0.2-0.8 \mathrm{eV}$ for L-FF and $0.4-1.3 \mathrm{eV}$ for D-FF, which is approximately 1.3-1.5 times higher for D-FF PNT than for L-FF (for the previous case of FF in the beta list it was $\sim 0.2 \mathrm{eV}[18,19])$ and, therefore, these PNT D-FF have a stronger and deeper interaction, which leads to a thicker growth of PNT D-FF as compared to L-FF. Note that the characteristic ratio of the diameters of the nanotubes obtained experimentally (in $\mu \mathrm{m}$ ) in [68] turns out to be 2.3/1.9 $\approx 1.21$, which is close to the ratio of their condensation energies established here. This latter conclusion is also confirmed by shorter distances between the DFF rings in the direction of the $a$-axis as compared to the L-FF rings: $\sim 23.8 \AA$ for D-FF and $24.8 \AA$ for L-FF (in experimental cases) and $\sim 28.8 \AA$ for D-FF and $29.0-29.8 \AA$ for L-FF for the cases of our models.

Therefore, this means that in this direction along $a$-axis, which is perpendicular to main tubular $c$-axis, the D-FF nanotubes have denser and stronger deep packing than L-FF PNT, and, therefore, more strongly adjacent nanotubes can adhere in this direction.

As result, D-FF PNT may increase to a greater width and thickness of the overall diameter of the nanotube bundle as compared to L-FF PNT. This effect was experimentally observed in recent works [68].

\subsection{Influence of topology and topological transition}

Second, another important conclusion is that all minimal energies for the optimal distances have significantly deeper values for the FF helix loop units obtained from experimentally created FF PNT, than for modeled ring structures. That means, that experimentally self-assembled PNT have more deeply optimized structures than artificially created and optimized PNT models based on rings. The main difference between simulated and experimentally created FF blocks is that the simulated FF structures have a more ordered model of FF rings, while FF blocks obtained from experimentally created FF structures have spiral (helical) structures. Moreover, the pitch of the helix (for each loop) has the value of the parameter of the crystallographic cell $c$ along the $c$-axis.

For this reason, the naturally-occurring optimized FF PNT structures have the lowest total energy per model unit or unit cell of a crystal. Consequently, in this experimental process of 
self-assembly of any FF PNT formation, such a nanotube goes through a naturally-occurring energy optimization. But this process is different for structures with different chirality in their original molecules and, as result, leads to the formation of two different helix structures within crystals with two types of enantiomers $-\mathrm{L}$ and $\mathrm{D}$, which have opposite changes in the order of chirality (or helicity), following the original molecular chirality of amino acids and dipeptides used. And this is characteristic of the general pattern of alternation of chirality with the complication of the hierarchy of molecular structures [28-30, 48-50].

Comparing the data obtained for the simulated and experimental structures, we can conclude that the energy of the experimental structures is lower, which means that these structures are better and deeper optimized. Consequently, such an experimental naturallyoccurring self-assembled optimization (it can be called self-optimization) under the experimental conditions led to more stable helix structures at the deepest minimum of energy.

As for artificially created and optimized structures that have not a helix coil, but only a ring structure, we must conclude that the energy optimization performed cannot reach such a deep minimum of energy. This result means that the helical structure is more preferable than the ring structure for these studied systems.

It should be emphasized that the optimization process performed by any algorithm is not so simple. It passes through a very complex potential energy surface (PES) for such polyatomic and many-electron systems, which have many local minima and various local energy barriers [110, 111]. Achieving some deeper and absolute energy minima for such a complex system is a very complex and lengthy task. In addition, the mathematical problems here are that, depending on the initial point and initial atomic configuration of the structure, it can go to a minimum along a different trajectory and, therefore, can occur at another point of local minima [110]. In any case, we can conclude that the optimization obtained using our algorithm on the structures of artificial models of the FF rings, as well as the FF PNT, may not be complete, terminating at some level of the local energy minimum.

But further steps of this optimization - from rings to helix loop - is very difficult, because it need to go through some yet unknown to us energy barrier. These two types of structures ring and helix - have different topology. Therefore, the transition from rings structure to helix structure is the topological transition. Recently such types of topological transitions have been in the focus of attention [110-113]. To explore this topological transition more thoroughly it is necessary to calculate full PES and transition path between ring and helix, going through some energy barrier. It is a very difficult and complex task and at the present moment we cannot evaluate it. But we can estimate the relative difference between the optimal energy states for these two types of topological structures, using our calculations results. This means that we can estimate the main energy of the topological transformation from a ring structure to a helix structure, which has the lower energy than that of the ring structure.

For different chiral cases and for various computational methods (quantum or molecular mechanical) we obtain the following energy values $E_{\mathrm{T}}$ of these topological transitions between ring and helix structures (with transformation along the main rotation $c$-axis):

- for the single ring-helix coil (or coil) transformation (by quantum PM3 RHF)

L-FF PNT: $E_{\mathrm{T}-\mathrm{L}}=-6.1795-(-1.014)=-5.1655 \mathrm{eV} \sim-5.17 \mathrm{eV}$,

D-FF PNT: $E_{\mathrm{T}-\mathrm{D}}=-6.22-(-0.607)=-5.613 \mathrm{eV} \sim-5.61 \mathrm{eV}$;

- for the single ring-helix turn (or coil) (by MM+ method)

L-FF PNT: ${ }^{\mathrm{M}-\mathrm{L}}=-2.732-(-1.759)=-0.973 \mathrm{eV}$,

D-FF PNT: $E_{\mathrm{T}-\mathrm{D}}^{\mathrm{M}}=-2.896-(-2.076)=-0.82 \mathrm{eV}$;

- for the double rings-turns (coils) transformation (by MM+ method)

L-FF PNT: $E_{\mathrm{T}-\mathrm{L}}^{\mathrm{Md}}=-2.508-(-1.40)=-1.108 \mathrm{eV}$,

D-FF PNT: $E_{\mathrm{T}-\mathrm{D}}^{\mathrm{Md}}=-3.265-(-1.81)=-1.455 \mathrm{eV}$. 
Thus, the energy of a given ring-helix topological transition is about $\sim 1-1.5 \mathrm{eV}$ when calculating the MM method, and about $\sim 5 \mathrm{eV}$, taking into account quantum effects.

These estimates of the energies of such a topological transition are preliminary and further development of this work is necessary. To compare these data more correctly we should probably estimate the specific transition energies per unit volume or cell. It is also important to take into account the configurational contribution and to estimate the change in the enthalpy and entropy during such transitions [31, 50, 110-112]. The approaches developed in $[112,114,115]$ on quantitative assessment of chirality in the hierarchies of protein structures and protein folding as an autowave process of self-organization in active media, on the development and study of patterns, concerning the spontaneous formation of LD-alternating hierarchies of chiral structures in biomacromolecules, initially non-equilibrium due to homochirality of primary structures, are also very important, interesting and promising for further investigations opening new insight of our knowledge of these living self-assembling processes. These areas are both fundamental and interdisciplinary, placed on the intersection of many sciences, and have numerous possibilities for a variety of practical applications.

It should be noted that the dipeptide L-FF can form another $\alpha$-helix structure - lefthanded (1-h)-helix [116]. In this case another dipole orientation of L-FF is obtained using HyperChem software (see Fig. 13 and Table 6). A discussion of the properties of such structures will be the subject of the following article.
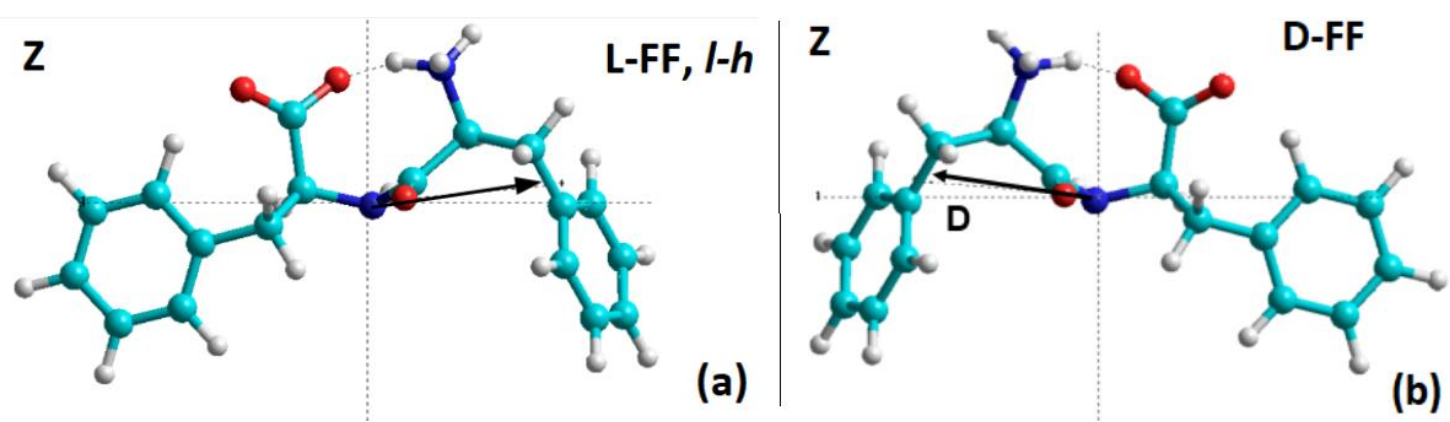

Fig. 13. Molecular models of diphenylalanine ( $\alpha$-helix conformation) in various isomer form: a) L-FF with chirality L and with left-handed (l-h)-helix, b) D-FF with chirality D.

Table 6. Data computed for dipeptides L-FF (1-h) and D-FF by PM3 method. Dipeptides consist from 43 atoms and have volume on the Van der Waals surface: $V_{\mathrm{L}-\mathrm{FF}, 1-\mathrm{h}}=291.919 \AA^{3}$, $\mathrm{V}_{\mathrm{D}-\mathrm{FF}}-291.977 \AA^{3}$

\begin{tabular}{|l|c|c|c|c|c|c|c|c|}
\multicolumn{1}{|c|}{ Method } & $\begin{array}{c}\mathrm{FF} \\
\text { chirality }\end{array}$ & $\begin{array}{c}D_{\mathrm{x}}, \\
\text { Debye }\end{array}$ & $\begin{array}{c}D_{\mathrm{y}}, \\
\text { Debye }\end{array}$ & $\begin{array}{c}D_{\mathrm{z}}, \\
\text { Debye }\end{array}$ & $\begin{array}{c}D_{\text {tot, }}, \\
\text { Debye }\end{array}$ & $\begin{array}{c}\text { Polarization, } \\
\mathrm{C} / \mathrm{m}^{2}\end{array}$ & $\begin{array}{c}\text { Total energy, } \\
\text { a.u. }\end{array}$ & $\begin{array}{c}\text { RMS } \\
\text { gradient }\end{array}$ \\
\hline $\begin{array}{l}\text { PM3, both } \\
\text { RHF and UHF, } \\
\text { 112 Orbitals }\end{array}$ & L-FF, 1-h & 11.645 & 1.115 & 0.899 & 11.733 & 0.13407 & -133.9591081 & $\sim 0.056$ \\
\cline { 2 - 9 } & D-FF & -11.63 & 1.052 & 1.113 & 11.73 & 0.134 & -133.959162 & $\sim 0.07$ \\
\hline
\end{tabular}

\section{CONCLUSIONS}

The results of this computational study compared with experimental data showed that the chirality of the original amino acids has a great influence on the following structure and properties of self-assembled tubular peptide structures based on their dipeptide molecules. In addition, self-assembled nanostructures obtained under natural conditions have more specific preferred topological helix structures than artificially optimized molecularly simulated analogous ring structures. In all the cases, the differences identified by the L-FF or D-FF chirality of these structures are significant and show the following differences in dipole moments, polarization, and the total energy of the systems. This dependence of properties on 
chirality is due to the different electrostatic dipole interactions of these different chiral molecular clusters. As a result of self-organization and self-assembly, the formation of FF PNT with different chirality has different structural features that determine the geometrical characteristics of the nanotubes themselves - their length, thickness and number of elementary nanotubes in one beam and energy properties (condensation energy).

One of the important problems is the fact that with the increasing complexity of hierarchical molecular systems, the chirality of such structures should change in accordance with the general rules of the basic biological system [28-30, 48-50]. This study confirms this recently established common property and opens up a new way for further study of the selforganization of biomolecular systems based on common rules and confirmed by computational molecular modeling using various methods. The results obtained in this study are very important for a more detailed interpretation of new experimental data and for further wide practical application.

In particular, it was found that the difference in chirality can significantly affect the processes of self-organization and self-assembly of various structures. Thus, with the FFbased PNT self-organization, it turned out that the condensation energy of the D-FF structural units is higher (and precisely in the transverse direction along the $a$-axis), and this leads to more efficient growth of these nanotubes in thickness - they are thicker and look like short bundles as compared with L-FF. The physical reason for such a difference can be the established difference in the dipole moments of the L-FF and D-FF dipeptide structures, which then manifests itself in their long-range electrostatic interaction and in more complex complexes and PNT structures. These results were obtained only by detailed modeling of these structures of different chirality and calculations of the features of their properties.

The important role of topology in the formation and self-assembly of such self-organizing complex molecular structures has also been established. In the process of their self-assembly, topological transitions can occur between structures of different levels of organization and corresponding topology. Such a topological transition occurs to provide the system with an energetically more favourable and optimal regime, as well as a new topological structure, which may be more stable in certain conditions.

The approach developed in this paper shows us further prospects in modeling and studying the features of the self-assembly processes of various self-organizing nanostructures, taking into account the possible influence of the chirality of the original amino acids on their properties. Of importance is also the role of the topology of individual structural components of the system and its changes. As a result, we get new information that is important for the development of our theoretical fundamental knowledge and new practical applications.

\section{ACKNOWLEDGEMENTS}

Part of this work was developed as part of the CICECO-Aveiro Materials Institute project, POCI-01-0145-FEDER-007679 funded from Fundação para a Ciência e a Tecnologia (FCT) Ref. UID/CTM/50011/2013, and funded from national funds through FCT/MEC, and cofunded by FEDER in accordance with the PT2020 Partnership Agreement. P.Z. thanks the project FCT PTDC/QEQ-QAN/6373/2014. S.K. thanks the project FCT PTDC/CTMCTM/31679/2017.

\section{REFERENCES}

1. Calvin M. Chemical evolution. Molecular evolution, towards the origin of living system on the Earth and elsewhere. Oxford: AT the Clarendon Press, 1969.

2. Lehninger A.L. Biochemistry. The molecular basis of cell structure and function. New York: Worth Publishers Inc., 1972.

3. Rees A.R., Sternberg M.J.E. From cells to atoms: an illustrated introduction to molecular biology. Blackwell Scientific Publications, 1984. 
4. Aryaa S.K., Solankia P.R., Dattab M., Malhotra B.D. Recent advances in selfassembled monolayers based biomolecular electronic devices. J. Biosensors and Bioelectronics. 2009. V. 24. № 9. P. 2810-2817.

5. Mendes A.C., Baran E.T., Reis R.L., Azevedo H.S. Self-assembly in nature: using the principles of nature to create complex nanobiomaterials. Wiley Interdiscip. Rev. Nanomed. Nanobiotechnol. 2013. V. 5. № 6. P. 582-612.

6. Orsi M. Molecular simulation of self-assembly. In: Self-assembling Biomaterials. $1^{\text {st }}$ Edition. Molecular Design, Characterization and Application in Biology and Medicine. Eds. Azevedo H.S., da Silva R.M.P. Elsevier Ltd. Woodhead Publishing, 2018. P. 305318. (Series in Biomaterials).

7. Lee O.S., Stupp S.I., Schatz G.C. Atomistic molecular dynamics simulations of peptide amphiphile self-assembly into cylindrical nanofibers. J. Am. Chem. Soc. 2011. V. 133. № 10. P. 3677-83.

8. Frith W.J. Self-assembly of small peptide amphiphiles, the structures formed and their applications. (A foods and home and personal care perspective). Philos. Trans. A. 2016. V. 374. № 2072. P. 2015-0138. doi: $10.1098 /$ rsta.2015.0138.

9. Van der Lit J., Marsman J.L., Koster R.S., Jacobse P.H., den Hartog S.A., Vanmaekelbergh D., Klein Gebbink R.J.M., Filion L., Ingmar Swart I.) Modeling the Self-Assembly of Organic Molecules in 2D Molecular Layers with Different Structures. J. Phys. Chem. C. 2016. V. 120. № 1. P. 318-323. doi: 10.1021/acs.jpcc.5b09889.

10. Brandon C.J., Martin B.P., McGee K.J., Stewart J.J.P., Braun-Sand S.B. An approach to creating a more realistic working model from a protein data bank entry. J. Mol. Mod. 2015. V. 21. P. 1-11.

11. Ghadiri M.R., Granja J.R., Milligan R.A., McRee D.E., Hazanovich N. Self assembling organic nanotubes based on cyclic peptide architecture. Nature. 1993. V. 366. P. 324327.

12. Görbitz C.H. Nanotube formation by hydrophobic dipeptides. Chem. Eur. J. 2001. V. 7. P. 5153-5159. doi: $10.1002 / 1521-3765(20011203) 7: 23<5153::$ AIDCHEM5153>3.0.CO;2-N.

13. Sedman V.L., Adler-Abramovich L., Allen S., Gazit E., Tendler S.J.B. Direct observation of the release of phenylalanine from diphenilalanine nanotubes. J. Am. Chem. Soc. 2006. V. 128. P. 6903-6908.

14. Scanlon S., Aggeli A. Self-assembling peptide nanotubes. Nano Today. 2008. V. 3. P. 22-30.

15. Shklovsky J., Beker P., Amdursky N., Gazit E., Rosenman G. Bioinspired peptide nanotubes: deposition technology and physical properties. Mater. Sci. Eng. B. 2010. V. 169. P. 62-66. doi: 10.1016/j.mseb.2009.12.040.

16. Bystrov V.S., Bdikin I., Heredia A., Pullar R.C., Mishina E., Sigov A., Kholkin A.L. Piezoelectricity and Ferroelectricity in biomaterials from proteins to self-assembled peptide nanotubes. In: Piezoelectric nanomaterials for biomedical applications. Eds. Ciofani G., Menciassi A. Berlin: Springer, 2012. P. 187-211.

17. Bystrov V.S., Seyedhosseini E., Kopyl S., Bdikin I.K., Kholkin A.L. Piezoelectricity and ferroelectricity in biomaterials: molecular modeling and piezoresponse force microscopy measurements. J. Appl. Phys. 2014. V. 116. № 6. P. 066803 . doi: 10.1063/1.4891443.

18. Bystrov V.S. Computer simulation nanostructures: bioferroelectric peptide nanotubes. Saarbrucken: LAP Lambert Academic Press, 2016. ISBN 978-3-659-92397-5.

19. Bystrov V.S., Paramonova E.V., Bdikin I.K., Kopyl S., Heredia A., Pullar R.C., Kholkin A.L. Bioferroelectricity: diphenylalanine peptide nanotubes computational modeling and ferroelectric properties at the nanoscale. Ferroelectrics. 2012. V. 440. № 1. P. 3-24. 
20. Nuraeva A., Vasilev S., Vasileva D., Zelenovskiy P., Chezganov D., Esin A., Kopyl S., Romanyuk K., Shur V.Ya., Kholkin A.L. Evaporation-Driven Crystallization of Diphenylalanine Microtubes for Microelectronic Applications. Cryst. Growth Des. 2016. V. 16. P. 1472-1479.

21. Weyl H. Symmetry. New Jersey, Princeton: Princeton University Press, 1952.

22. Kane G. Supersymmetry and Beyond: From the Higgs Boson to the New Physics. Basic Books, 2013. 216 p.

23. Feynman R. The Character of Physical Law. 1965. 173 p.

24. Chirality and Biological Activity. Eds. Holmstedt B., Frank H., Testa B. New York: Liss, 1990.

25. IUPAC. Compendium of Chemical Terminology. Compiled by McNaught A.D., Wilkinson A. Oxford: Blackwell Scientific Publications, 1997.

26. Capito R.M., Azevedo H.S., Velichko Y.S., Mata A., Stupp S.I. Self-assembly of large and small molecules into hierarchically ordered sacs and membranes. Science. 2008. V. 319. № 5871. P. 1812-1816. doi: 10.1126/science.1154586.

27. Yashima E., Ousaka N., Taura D., Shimomura K., Ikai T., Maeda K. Supramolecular Helical Systems: Helical Assemblies of Small Molecules, Foldamers, and Polymers with Chiral Amplification and Their Functions. Chem. Rev. 2016. V. 116. № 22. P. 13752-13990. doi: 10.1021/acs.chemrev.6b00354.

28. Tverdislov V.A. Chirality as a primary switch of hierarchical levels in molecular biological systems. Biophysics. 2013. V. 58. № 1. P. 128-132.

29. Malyshko E.V., Tverdislov V.A. IOP. J. Phys. Conf. Series. 2016. V. 741. P. 012065. doi: 10.1088/1742-6596/741/1/012065.

30. Tverdislov V.A., Malyshko E.V., Il'chenko S.A., Zhulyabina O.A., Yakovenko L.V. A periodic system of chiral structures in molecular biology. Biophysics. 2017. V. 62. № 3. P. 331-341. doi: 10.1134/S0006350917030228.

31. Cantor Ch.R., Schimel P.R. Biophysical Chemistry. Part 3. The Behavior of Biological Molecules. San Francisco: W.H. Freeman and Company, 1980.

32. Eliel E.L., Wilen S., Doyle M. Basic Organic Stereochemistry. New York: WileyInterscience, 2001.

33. Müller U. Symmetry Relationships between Crystal Structures. Applications of Crystallographic Group Theory in Crystal Chemistry. Oxford: University Press, 2013.

34. Lam H., Oh D.C., Cava F., Takacs C.N., Clardy J., de Pedro M.A., Waldor M.K. Damino acids govern stationary phase cell wall remodeling in bacteria. Science. 2009. V. 325. № 5947. P. 1552-1555. doi: 10.1126/science.1178123.

35. Tishkov V. I. The Coenzyme Regeneration for Biosynthesis of Chiral Compounds Using Dehydrogenases. Moscow University Chemistry Bulletin. 2002. V. 43. № 6. P. 381-388 (in Russ.).

36. Mason S.F. Origins of biomolecular handedness. Nature. 1984. V. 311. P. 19-23.

37. Blanke S.R. Expanding Functionality within the Looking-Glass Universe. Science. 2009. V. 325. P. 1505-1506.

38. Verbiest T, Van Elshocht S., Kauranen M., Hellemans L., Snauwaert J., Nuckolls C., Katz T.J., Persoons A. Strong Enhancement of Nonlinear Optical Properties Through Supramolecular Chirality. Science. 1998. V. 282. P. 913-915. doi: 10.1126/science.282.5390.913.

39. Naaman R., Waldeck D.H. Chiral-Induced Spin Selectivity Effect. J. Phys. Chem. Lett. 2012. V. 3. № 16. P. 2178-2187. doi: 10.1021/jz300793y.

40. Naaman R., Waldeck D.H. Spintronics and Chirality: Spin Selectivity in Electron Transport Through Chiral Molecules. Annu. Rev. Phys. Chem. 2015. V. 66. P. 263-81. 
41. Dreiling J.M., Gay T.J. Chirally Sensitive Electron-Induced Molecular Breakup and the Vester-Ulbricht Hypothesis. Phys. Rev. Lett. 2014. V. 113. P. 118103. doi: 10.1103/PhysRevLett.113.118103.

42. Ulbricht T.L.V., Vester F. Attempts to induce optical activity with polarized b-radiation. Tetrahedron. 1962. V. 18. №. 5. P. 629-637. doi: 10.1016/S0040-4020(01)92714-0.

43. Sokolov A.A., Ternov I.M. On Polarization and Spin Effects in Theory of Synchrotron Radiation. Sov. Phys. Dokl. 1964. V. 8. P. 1203.

44. Ternov I.M., Bagrov V.G., Rzaev R.A. Izvestiia VUZov. Fizika (Russian Physics Journal). 1963. V. 5. P. 127-139 (in Russ.).

45. Kettner M., Göhler B., Zacharias H., Mishra D., Kiran V., Naaman R., Fontanesi C., Waldeck D.H., Sęk S., Pawłowski J., Juhaniewicz J. Spin Filtering in Electron Transport Through Chiral Oligopeptides. J. Phys. Chem. C. 2015. V. 119. P. 14542-14547. doi: 10.1021/jp509974z.

46. Gohler B., Hamelbeck V., Markus T.Z., Kettner M., Hanne G.F., Vager Z., Naaman R., Zacharias H. Spin Selectivity in Electron Transmission Through Self-Assembled Monolayers of Double-Stranded DNA. Science. 2011. V. 331. P. 894-897. doi: $10.1126 /$ science.1199339.

47. Banerjee-Ghosh K., Dor O.B., Tassinari F., Capua E., Yochelis S., Capua A., Yang S.-H., Parkin S.S.P., Sarkar S., Kronik L., Baczewski L.T., Naaman R., Paltiel Y. Separation of enantiomers by their enantiospecific interaction with achiral magnetic substrates. Science. 2018. V. 360. № 6395. P. 1331-1334. doi: 10.1126/science.aar4265.

48. Zhuliabina O.A., Malyshko E.V., Il'chenko S.A., Tverdislov V.A. Vestnik nauki $i$ obrazovaniia (Bulletin of science and education). 2015. V. 4. № 6. C. 24-25 (in Russ.).

49. Malyshko E.V., Tverdislov V.A. Physical Principles of Discrete Hierarchies Formation in Protein Macromolecules. IOP Conf. Series: Journal of Physics: Conf. Serie. 2017. V. 917. P. 42025.

50. Malyshko E.V. Khiral'nyi dualizm kak fizicheskaia osnova stratifikatsii v strukturnykh ierarkhiiakh belkov (Chiral dualism as the physical basis of stratification in protein structural hierarchies): Ph. D. Thesis. Moscow, 2018. 146 p. (in Russ.).

51. Kharzeev D.E. The Chiral Magnetic Effect and Anomaly-Induced Transport. Prog. Part. Nucl. Phys. 2014. V. 75. P. 133-151. doi: 10.1016/j.ppnp.2014.01.002.

52. Hirono Yu., Kharzeev D.E., Sadofyev A.V. Dynamics of vortices in chiral media: the chiral propulsion effect. Phys. Rev. Lett. 2018. V. 121. № 14. P. 142301. doi: 10.1103/PhysRevLett.121.142301.

53. Kaushik S., Kharzeev D.E. Quantum Oscillations in the Chiral Magnetic Conductivity. Phys. Rev. B. 2017. V. 95. № 23. P. 235136. doi: 10.1103/PhysRevB.95.235136.

54. Cortijo A., Kharzeev D., Landsteiner K., Vozmediano M.A.H. Strain induced Chiral Magnetic Effect in Weyl semimetals. Phys. Rev. B. 2016. V. 94. № 24. P. 24140. doi: 10.1103/PhysRevB.94.241405.

55. Yin P., Zhang Z.M., Lv H., Li T., Haso F., Hu L., Zhang B., Bacsa J., Wei Y., Gao Y., Hou Y., Li Y.G., Hill C.L., Wang E.B., Liu T. Chiral recognition and selection during the self-assembly process of protein-mimic macroanions. Nature Comm. 2015. V. 6 P. 6475. doi: $10.1038 /$ ncomms7475.

56. Kanduc M., Dobnikar J., Podgornik R. Counterion-mediated electrostatic interactions between helical molecules. Soft Matter. 2009. V. 5. P. 868-877. doi: 10.1039/b811795k.

57. Bystrov V.S., Singh B., Bdikin I.K., Tverdislov V.A., Zhulyabina O.A. Ferroelectric physical properties of various dipeptide nanotubes. In: International Conference on Nanomaterials Science and Mechanical Engineering (University of Aveiro, Portugal, July 16-18, 2018): Book of Abstracts. Eds. Bdikin I., Neto V.F.S. Aveiro: UA Editora, Universidade de Aveiro, 2018. P. 62. 
58. Bdikin I., Singh B., Bystrov V.S. Local piezoelectricity in amino acids microcrystals. In: International Conference on Nanomaterials Science and Mechanical Engineering (University of Aveiro, Portugal, July 16-18, 2018): Book of Abstracts. Eds. Bdikin I., Neto V.F.S. Aveiro: UA Editora, Universidade de Aveiro, 2018. P. 78.

59. Kopyl S., Bystrov V.S., Nuraeva A., Zelenovskiy P., Vasilev S., Arkhipov S.G., Shur V.Ya., Kholkin A.L. Diphenylalanine Peptide Nanotubes with Different Chirality: Structures, Properties, and Applications. In: International Conference on Nanomaterials Science and Mechanical Engineering (University of Aveiro, Portugal, July 16-18, 2018): Book of Abstracts. Eds. Bdikin I., Neto V.F.S. Aveiro: UA Editora, Universidade de Aveiro, 2018. P. 41.

60. Bystrov V.S., Bdikin I.K., Tverdislov V.A., Zhulyabina O.A., Zelenovskiy P.S., Kopyl S.A. Physical ferroelectric and chiral properties of various dipeptide nanotubes and nanostructures. In: Proceedings of the International Conference "Mathematical Biology and Bioinformatics". Ed. V.D. Lakhno. Vol. 7. Pushchino: IMPB RAS, 2018. Paper No. e9. doi: 10.17537/icmbb18.4.

61. Reches M.,Gazit E. Controlled patterning of aligned self-assembled peptide nanotubes. Nature Nanotech. 2006. V. 1. P. 195-200.

62. Adler-Abramovich L., Aronov D., Beker P., Yevnin M., Stempler S., Buzhansky L., Rosenman G., Gazit E. Self-assembled arrays of peptide nanotubes byvapour deposition. Nature Nanotechnology. 2009. V. 4. P. 849-854.

63. Adler-Abramovich L., Gazit E. The physical properties of supramolecular peptide assemblies: from building block association to technological application. Chem. Soc. Rev. 2014. V. 43. P. 6881-6893.

64. Amdursky N., Molotskii M., Aronov D., Adler-Abramovich L., Gazit E., Rozenman G. Blue luminescence based on quantum confinement at peptide nanotubes. Nano Letters. 2009. V. 9. № 9. P. 3111-3115.

65. Kol N., Adler-Abramovich L., Barlam D., Shneck R.Z., Gazit E., Rousso I. Selfassembled peptide nanotubes are uniquely rigid bioinspired supramolecular structures. Nano Lett. 2005. V. 5. P. 1343-1346.

66. Zelenovskiy P., Kornev I., Vasilev S., Kholkin A. On the origin of the great rigidity of self-assembled diphenylalanine nanotubes. Phys. Chem. Chem. Phys. 2016. V. 18. № 43. P. 29681-29685.

67. Zelenovskiy P.S., Davydov A.O., Krylov A.S., Kholkin A.L. Raman study of structural transformations in self-assembled diphenylalanine nanotubes at elevated temperatures. J. Raman Spectrosc. 2017. V. 48. № 11. P. 1401-1405.

68. Zelenovskiy P.S., Nuraeva A.S., Kopyl S., Arkhipov S.G., Vasilev S.G., Bystrov V.S., Svitlyk V., Shur V.Ya., Mafra L., Kholkin A.L. Chirality-dependent growth of selfassembled diphenylalanine microtubes. Phys. Chem. Chem. Phys. 2019.

69. The Cambridge Crystallographic Data Centre (CCDC). URL: https://www.ccdc.cam.ac.uk/ (accessed 23.01.2019).

70. Bystrov V.S., Kopyl S.A., Zelenovskiy P., Zhulyabina O.A., Tverdislov V.A., Salehli F., Ghermani N.E., Shur V.Ya., Kholkin A.L. Investigation of physical properties of diphenylalanine peptide nanotubes having different chiralities and embedded water molecules. Ferroelectrics. 2018. V. 525. P. 168-177. doi: 10.1080/00150193.2018.14328.

71. HyperChem Professional 8.0. URL: http://www.hyper.com/?tabid=360 (accessed 20.02.2019).

72. Stewart J.J.P. Optimization of Parameters for Semiempirical Methods. I. Method. J. Comput. Chem. 1989. V. 10. P. 209. 
73. Stewart J.J.P. Optimization of parameters for semiempirical methods V: Modification of NDDO approximations and application to 70 elements. J. Mol. Mod. 2007. V. 13. № 12. P. $1173-1213$.

74. Szabo A., Ostlund N. Modern Quantum Chemistry. New York: Macmillan, 1985.

75. Clark T.A. Handbook of Computational Chemistry. New York: John Wiley and Sons, 1985.

76. Kohn W., Sham L.J. Self-Consistent Equations Including Exchange and Correlation Effects. Phys. Rev. 1965. V. 140. P. A1133.

77. Kresse G., Hafner J. Ab initio. Phys. Rev. B. 1994. V. 49. P. 14251-14269.

78. Kresse G., Furthmüller J. Efficient iterative schemes for ab initio total-energy calculations using a plane-wave basis set. Phys. Rev. B. 1996. V. 54. P. 11169-11186.

79. Kresse G., Joubert D. From ultrasoft pseudopotentials to the projector augmented wave method. Physical Review B. 1999. V. 59. P. 1758-1775.

80. Perdew J.P., Burke K., Ernzerhof M. Generalized Gradient Approximation Made Simple. Phys. Rev. Lett. 1996. V. 77. P. 3865-3868.

81. Lee C., Yang W., Parr R.G. Development of the Colle-Salvetti correlation-energy formula into a functional of the electron density. Phys. Rev. B. 1988. V. 37. P. 785-789.

82. Becke A.D. A new mixing of Hartree-Fock and local density-functional theories. $J$. Chem. Phys. 1993. V. 98. P. 1372-1377.

83. Pople J.A., Beveridge D.L. Approximate Molecular Orbital Theory. New York: McGraw-Hill, 1970.

84. Krishnan R., Kinkley J.S., Seeger R., Pople J.A. Self-consistent molecular orbital methods. XX. A basis set for correlated wave functions. J. Chem. Phys. 1980. V. 72. P. 650-654.

85. McLean A.D., Chandler G.S. Contracted Gaussian basis sets for molecular calculations. I. Second row atoms, Z=11-18. J. Chem. Phys. 1980. V. 72. P. 5639-5648.

86. Møller Ch., Plesset M. Note on an Approximation Treatment for Many-Electron Systems. Phys. Rev. 1934. V. 46. № 7. P. 618-622.

87. Head-Gordon M., Pople J.A., Frisch M.J. MP2 energy evaluation by direct methods. Chem. Phys. Lett. 1988. V. 153. № 6. P. 503-506.

88. Hamprecht F.A., Cohen A.J., Tozer D.J., Handy N.C. Development and Assessment of New Exchange-correlation Functionals. J. Chem. Phys. 1998. V. 109. P. 6264.

89. Bystrov V.S., Zelenovskiy P.S., Nuraeva A.S., Kopyl S.A., Zhulyabina O.A., Tverdislov V.A. Molecular modeling and computational study of the chiral-dependent structures and properties of the self-assembling diphenylalanine peptide nanotubes. $J$. Mol. Mod. 2019.

90. Bystrov V.S., Paramonova E.V., Dekhtyar Yu., Pullar R.C., Katashev A., Polyaka N., Bystrova A.V., Sapronova A.V., Fridkin V.M., Kliem H., Kholkin A.L. Polarization of poly(vinylidene fluoride) and poly(vinylidene fluoridetrifluoroethylene) thin films revealed by emission spectroscopy with computational simulation during phase transition. J. Appl. Phys. 2012. V. 111. P. 104113. doi: 10.1063/1.4721373.

91. Bystrov V.S., Paramonova E.V., Bdikin I.K., Bystrova A.V., Pullar R.C., Kholkin A.L. Molecular modeling of the piezoelectric effectin the ferroelectric polymer poly(vinylidene fluoride) (PVDF). J. Mol. Model. 2013. V. 19. P. 3591-3602. doi: $10.1007 / \mathrm{s} 00894-013-1891-\mathrm{z}$.

92. Bystrov V.S., Bdikin I.K., Silibin M., Karpinsky D., Kopyl S., Paramonova E.V., Goncalves G. Molecular modeling of the piezoelectric properties of ferroelectric composites containing polyvinylidene fluoride (PVDF) and either graphene or graphene oxide. J. Mol. Mod. 2017. V. 23. № 4. P. 128. doi: 10.1007/s00894-017-3291-2.

93. Murrell J.N., Harget A.J. Semi-empirical Self-consistent-field Molecular Orbital Theory of Molecules. New York: Wiley Interscience, 1971. 
94. Brandon C.J., Martin B.P., McGee K.J., Stewart J.J.P., Braun-Sand S.B. An approach to creating a more realistic working model from a protein data bank entry. J. Mol. Mod. 2015. V. 21. № 1. P. 11.

95. Dewar M.J.S., Thiel W. The MNDO method. Approximations and parameters. J. Amer. Chem. Soc. 1977. V. 99. 4899-4906.

96. Stewart J.J.P. An investigation into the applicability of the semiempirical method PM7 for modeling the catalytic mechanism in the enzyme Chymotrypsin. J. Mol. Mod. 2017. V. 23. P. 154. doi: 10.1007/s00894-017-3326-8.

97. Dewar M.J.S., Zoebisch E.G., Healy E.F., Stewart J.J.P. A new general purpose quantum mechanical molecular model. J. Am. Chem. Soc. 1985. V. 107. P. 3902-3909.

98. Andrade-Filho T., Martins T.C., Ferreira F.F., Alves W.A., Rocha A.R. Water-driven stabilization of diphenylalanine nanotube structures. Theor. Chem. Acc. 2016. V. 135. № 8. P. 185. doi: 10.1007/s00214-016-1936-3.

99. Klein E., Matis M., Lukes V., Cibulkova Z. The applicability of AM1 and PM3 semiempirical methods for the study of $\mathrm{NeH}$ bond dissociation enthalpies and ionisation potentials of amine type antioxidants. Polymer Degradation and Stability. 2006. V. 91. P. 262-270.

100. Allinger N.L. Conformational analysis. 130. MM2. A hydrocarbon force field utilizing V1 and V2 torsional terms. J. Am. Chem. Soc. 1977. V. 99. № 25. P. 8127-8134. doi: 10.1021/ja00467a001.

101. Weiner S.J., Kollman P.A., Case D.A., Singh U.C., Ghio C., Alagona G., Profeta Jr.S., Weiner P. A new force field for molecular mechanical simulation of nucleic acids and proteins. J. Am. Chem. Soc. 1984. V. 106. P. 765-784.

102. Weiner S.J., Kollman P.A., Nguyen D.T., Case D.A. An all atom force field for simulations of proteins and nucleic acids J. Comput. Chem. 1986. V. 7. P. 230-252.

103. Cornell W.D., Cieplak P., Bayly C.I., Gould I.R., Merz K.M.Jr., Ferguson D.M., Spellmeyer D.C., Fox T., Caldwell J.W., Kollman P.A. A second generation force field for the simulation of proteins and nucleic acids. J. Am. Chem. Soc. 1995. V. 117. P. 5179-5197.

104. Brooks B.R., Bruccoleri R.E., Olafson B.D., States D.J., Swaminathan S., Karplus M. CHARMM: A program for macromolecular energy, minimization, and dynamics calculations. J. Comput. Chem. 1983. V. 4. P. 187-217.

105. Flack H.D., Bernardinelli G. The use of X-ray crystallography to determine absolute configuration. Chirality. 2008. V. 20. P. 681-690.

106. Flack H.D. The use of X-ray Crystallography to Determine Absolute Configuration (II). Acta Chim. Slov. 2008. V. 55. № 4. P. 689-691.

107. International Tables for Crystallography. Volume A: Space-Group Symmetry. Ed. Hahn Th. Netherlands: Spriger, 2005.

108. Open Babel. URL: https://openbabel.org/docs/dev/Installation/install.html (accessed 20.02.2019).

109. Lennard-Jones J.E. On the Determination of Molecular Fields. Proc. R. Soc. Lond. A. 1924. V. 106. № 738. P. 463-477.

110. Shaitan R.V., Lozhnikov M.A., Kobelkov G.M. Relaxation Folding and the Principle of the Minimum Rate of Energy Dissipation for Conformational Motions in a Viscous Medium. Biophysics. 2016. V. 61. № 4. P. 531-538.

111. Grosberg A.Y. Khokhlov A.R. Giant Molecules: Here, There, and Everywhere. Academic Press, 1997. ISBN 0-12-304130-9.

112. Sidorova A.E., Malyshko E.V., Kotov A.R., Levashova N.T., Ustinin M.N., Tverdislov V.A. Protein Folding as an Autowave Process of Self-Organization in Active Media. Bulletin of the Russian Academy of Sciences: Physic. 2019. V. 83. № 1. P. 85-90. 
113. Hadzibabic Z., Kruger P., Cheneau M., Battelier B., Dalibard J. Berezinskii-KosterlitzThouless crossover in a trapped atomic gas. Nature. 2006. V. 441. P. 1118-1121.

114. Sidorova A.E., Tverdislov V.A., Levashova N.T., Kotov A.R., Ustinin M.N. Quantitative approach to the assessment of chirality in hierarchies of protein structures. In: Proceedings of the International Conference "Mathematical Biology and Bioinformatics". Ed. V.D. Lakhno. Vol. 7. Pushchino: IMPB RAS, 2018. Paper No. e82. doi: 10.17537/icmbb18.96.

115. Tverdislov V.A., Malyshko E.V. Chiral dualism, arrow of symmetry and molecular machines. In: Proceedings of the International Conference "Mathematical Biology and Bioinformatics". Ed. V.D. Lakhno. Vol. 7. Pushchino: IMPB RAS, 2018. Paper No 81. doi: $10.17537 / \mathrm{icmbb} 18.97$.

116. Novotny M., Kleywegt G.J. A survey of left-handed helices in protein structures. J. Mol. Biol. 2005. V. 347. № 2. P. 231-410. doi: 10.1016/j.jmb.2005.01.037. 